\title{
ac Susceptometry of 2D van der Waals Magnets Enabled by the Coherent Control of Quantum Sensors
}

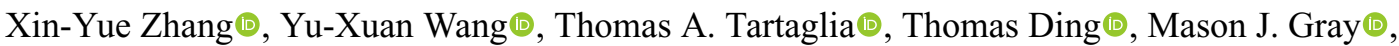 \\ Kenneth S. Burch®, Fazel Tafti®, and Brian B. Zhou®* \\ Department of Physics, Boston College, Chestnut Hill, Massachusetts 02467, USA
}

(Received 17 May 2021; accepted 23 August 2021; published 28 September 2021)

\begin{abstract}
Precision magnetometry is fundamental to the development of novel magnetic materials and devices. Recently, the nitrogen-vacancy (NV) center in diamond has emerged as a promising probe for static magnetism in two-dimensional (2D) van der Waals materials, capable of quantitative imaging with nanoscale spatial resolution. However, the dynamic character of magnetism, crucial for understanding the magnetic phase transition and achieving technological applications, has rarely been experimentally accessible in single 2D crystals. Here, we coherently control the NV center's spin precession to achieve ultrasensitive, quantitative ac susceptometry of a 2D ferromagnet. Combining dc hysteresis with ac susceptibility measurements varying temperature, field, and frequency, we illuminate the formation, mobility, and consolidation of magnetic domain walls in few-layer $\mathrm{CrBr}_{3}$. We show that domain wall mobility is enhanced in ultrathin $\mathrm{CrBr}_{3}$, with minimal decrease for excitation frequencies exceeding hundreds of kilohertz, and is influenced by the domain morphology and local pinning of the flake. Our technique extends NV magnetometry to the multifunctional ac and dc magnetic characterization of wide-ranging spintronic materials at the nanoscale.
\end{abstract}

DOI: 10.1103/PRXQuantum.2.030352

\section{INTRODUCTION}

Two-dimensional van der Waals (vdW) magnetic materials have broached transformative concepts for integrating and controlling spintronic devices, but also presented unique challenges to their magnetic characterization [1-3]. Notably, the magnetic moment of a micron-sized exfoliated monolayer (approximately $10^{-11} \mathrm{emu}$ ) lies below the sensitivity (approximately $10^{-8} \mathrm{emu}$ ) of commercial magnetometers based on the superconducting quantum interference device (SQUID) [4], the benchmark for analyzing bulk magnetic materials. Key insights in vdW magnetism are most frequently provided by magneto-optical [5-8] and photoluminescence (PL) imaging $[9,10]$, which feature high sensitivity, or by electrical measurements, which directly incorporate layers inside functional devices [11-13]. However, these approaches depend sensitively on material- and device-specific coupling of the sample's magnetization to the measured optical or transport

\footnotetext{
*brian.zhou@bc.edu
}

Published by the American Physical Society under the terms of the Creative Commons Attribution 4.0 International license. Further distribution of this work must maintain attribution to the author(s) and the published article's title, journal citation, and DOI. properties, which complicates quantitative interpretation and restricts generalizability [14-16]. A direct probe of the magnetic field associated with both static and dynamic magnetizations in two-dimensional (2D) crystals would accelerate materials discovery and optimization.

Detecting stray fields from single 2D magnetic flakes is challenging due to the requirement of miniaturized sensors that must be deployed in nanoscale proximity to these microscopic samples. Pioneering efforts have utilized mesoscopic graphene-based Hall bars [17] and atomicscale spin defects in diamond [18-21] to measure static stray fields and quantify the absolute magnetization of mono- and few-layer ferromagnets. However, the dynamic signatures of 2D magnets [7,8,11,22], which predominate near magnetic criticality, have yet to be investigated directly through magnetometry. In particular, ac susceptibility, which probes the response of the sample to an oscillating magnetic field, is a powerful technique for understanding magnetic phase transitions, relaxation times, and domain dynamics in diverse materials, including antiferromagnets, spin glasses, and ensembles of single-molecule magnets $[23,24]$. At the Curie temperature $\left(T_{c}\right)$ of the paramagnetic to ferromagnetic phase transition, the ac susceptibility $\chi_{\mathrm{ac}}$ diverges with a critical exponent $\gamma$ characteristic of the universality class of the underlying interactions [25]. Below $T_{c}, \chi_{\mathrm{ac}}$ senses magnetization rotation, domain wall motion, superparamagnetism, and the 
interactions of these processes with defects, strain, and external dc field [26].

In this work, we leverage the coherent manipulation of quantum sensors to probe both the dc and ac magnetic properties of a $2 \mathrm{D} \mathrm{vdW}$ magnet. We exfoliate ultrathin flakes of the ferromagnetic insulator $\mathrm{CrBr}_{3}$ onto a diamond magnetometer chip containing a near-surface ensemble of nitrogen-vacancy (NV) centers. This versatile platform directly enables sensitive measurement of both the 2D ferromagnet's dc magnetization through static shifts of the NV center's spin energies [18-21] and its ac susceptibility through dynamically coupling the NV center's spin precession to ac fields using frequency-selective quantum control sequences $[27,28]$. Our dc measurements of magnetic hysteresis reveal that the details of domain nucleation and pinning can differ significantly in ultrathin, exfoliated $\mathrm{CrBr}_{3}$ flakes of similar thickness (approximately ten layers), pointing to large variability in the impact of the local microstructure. In complement, ac susceptibility measurements on few-layer $\mathrm{CrBr}_{3}$ indicate a critical exponent $\gamma=$ $1.1 \pm 0.3$ for the ferromagnetic phase transition and display distinct features that illuminate the formation, mobility, and consolidation of single domain walls below $T_{c}$, informing on the potential for domain-based memory and logic devices [29-31]. Our work achieves an ac field resolution of approximately $40 \mathrm{nT}$, representing the most sensitive NV magnetometry performed to date on exfoliated 2D magnets [18-21], and introduces a generic platform for understanding subgigahertz dynamical phenomena in $2 \mathrm{D}$ magnetism.

\section{MAGNETIC PROPERTIES MEASUREMENT SYSTEM FOR 2D vdW MAGNETS}

Figure 1(a) displays our experimental platform based on the optical readout of a layer of near-surface NV center spins (approximately $60 \mathrm{~nm}$ depth). We exfoliate $\mathrm{CrBr}_{3}$ flakes [32,33] without encapsulation onto a diamond substrate inside an argon-filled glovebox and then transfer the substrate into our cryostat with minimized exposure to ambient (see Appendix A for experimental details). An insulated wire coil adjacent to the diamond delivers both the microwave pulses (order of gigahertz) for NV center spin manipulation and the radio frequency (order of $100 \mathrm{kHz}$ ) excitation field $\vec{B}_{\text {ac }}^{C}$ for probing the ac response of the ferromagnet. In the main text, we use $\vec{B}$ to denote the vector magnetic field (e.g., $\vec{B}_{\text {ac }}^{C}$ ), and $B$ to denote its projection onto the NV center axis (e.g., $B_{\mathrm{ac}}^{C}$ ). The external magnetic field $\vec{B}_{\mathrm{dc}}^{E}$ is carefully aligned along one of the four NV center crystallographic orientations, forming an angle $\theta=54.7^{\circ}$ to the surface normal. Away from $T_{c}$, the applied, in-plane component of $\vec{B}_{\mathrm{dc}}^{E}$ in our experiments $(<0.05 \mathrm{~T})$ can be neglected as $\mathrm{CrBr}_{3}$ possesses a large uniaxial anisotropy that pins the magnetization $M$ to the out-of-plane direction ( $z$ axis) [34]. (a)
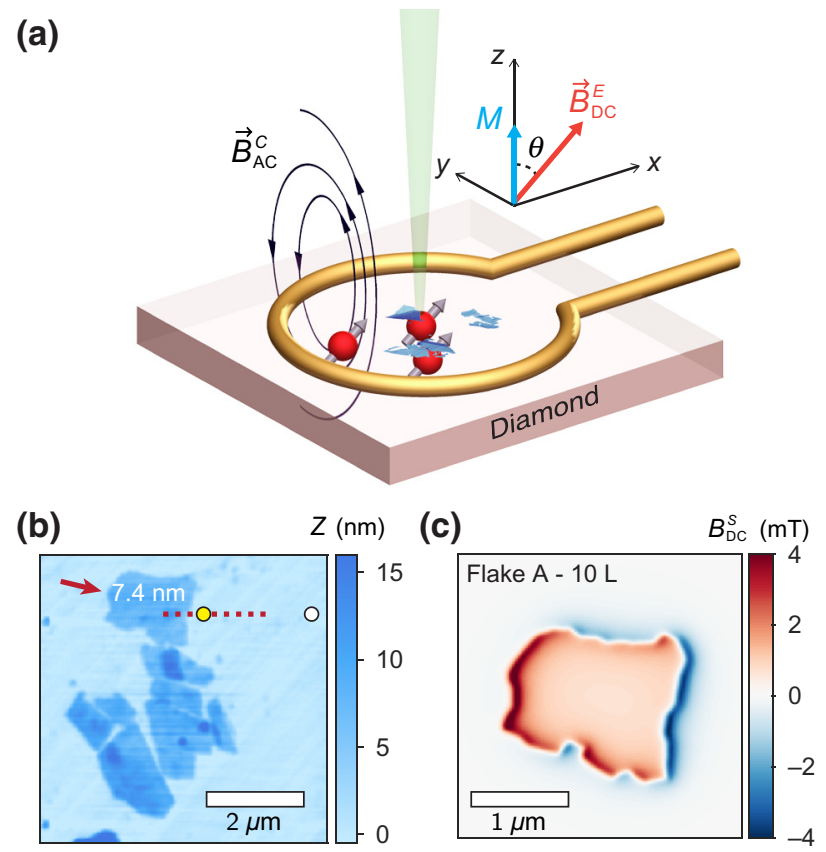

FIG. 1. Experimental overview. (a) $\mathrm{CrBr}_{3}$ flakes (blue) are exfoliated onto a diamond chip containing a shallow ensemble of NV centers (red). A coil delivers both the ac field $\vec{B}_{\mathrm{ac}}^{C}$ for exciting the magnetic dynamics of the ferromagnet and the gigahertzfrequency pulses to manipulate the NV center spin. The static bias field $\vec{B}_{\mathrm{dc}}^{E}$ is aligned along the NV center axis, while the easy axis for the magnetization $M$ is along the out-of-plane, $z$ direction. (b) AFM image of Flake A (arrow), ten layers thick. Measurements are primarily taken outside the flake's right edge (yellow dot) and are referenced to the background signal far away from the flake (e.g., white dot). (c) Simulated stray field map for Flake A, assuming a NV center depth of $60 \mathrm{~nm}$. Here $B_{\mathrm{dc}}^{S}$ is the component of the flake's stray field parallel to the NV center axis.

We identify $\mathrm{CrBr}_{3}$ flakes as thin as six layers for low-temperature measurement by their optical contrast on diamond. The thicknesses of the investigated flakes are subsequently verified by atomic force microscopy (AFM), as exemplified in Fig. 1(b), showing an image of a 7.4nm-thick $\mathrm{CrBr}_{3}$ flake (Flake A). In Fig. 1(c), we perform simulations of Flake A's stray field at $60 \mathrm{~nm}$ beneath the diamond surface [18]. We assume a saturated magnetic moment density of $148 \mu_{b} / \mathrm{nm}^{2}$ (3.0 $\mu_{b}$ per Cr ion) for ten layers with ferromagnetic interlayer coupling and plot $B_{\mathrm{dc}}^{S}$, the projection of the sample's stray field onto the oblique NV center axis. In this work, we utilize the $\left|m_{S}=0\right\rangle \rightarrow$ $\left|m_{S}=-1\right\rangle$ transition in the NV center ground state, which disperses with a slope $-\gamma_{e} / 2 \pi=-28 \mathrm{MHz} / \mathrm{mT}$ [28]. Hence, outside the right edge of the flake, where many of our measurements are performed, we expect a strong positive (negative) Zeeman shift of the NV center's transition frequency if the flake is magnetized parallel (antiparallel) to the component of $\vec{B}_{\mathrm{dc}}^{E}$ along the $z$ axis. 


\section{III. de FIELD SENSING OF MAGNETIC HYSTERESIS}

In Fig. 2(a), we present the optically detected magnetic resonance (ODMR) spectra at $4 \mathrm{~K}$ for a linecut across the right edge of another $\mathrm{CrBr}_{3}$ flake (Flake $\mathrm{B}$, $6.7 \mathrm{~nm} / 9$ layers) with a long, straight edge (see Fig. 8 for AFM image). Approaching the right edge of the flake from the outside $(x>0)$, the NV magnetic resonance shifts to higher frequencies, before switching to lower frequencies in the interior of the flake $(x<0)$, corroborating a ferromagnetic moment aligned with the out-of-plane component of $\vec{B}_{\mathrm{dc}}^{E}$. In comparison to single NV measurements [18], we observe broadening of the ensemble NV center linewidth due to spatial averaging over strong magnetic field gradients near the flake's edge.

The evolution of the lineshape, however, can be accurately modeled [solid lines in Fig. 2(a)] by summing intrinsic Lorentzian lineshapes with a distribution of center frequencies (see Appendix C). For each center location $x$ of the optical spot, we determine the distribution of stray fields $B_{\mathrm{dc}}^{S}$ within the spot [Fig. 2(b)] by assuming a Gaussian beam shape (standard deviation $\sigma_{B}=170$ $\mathrm{nm}$ ) and a stray field profile corresponding to the edge of a semi-infinite magnetic sheet [35]. Fitting the lineshapes simultaneously using this model, we extract the magnetization of Flake B to be $86 \pm 5 \mu_{b} / \mathrm{nm}^{2}$ at $B_{\mathrm{dc}}^{E}=$
$76 \mathrm{mT}$. This value is lower than expected for nine-layer $\mathrm{CrBr}_{3}$ at saturation $\left(133 \mu_{b} / \mathrm{nm}^{2}\right)$. Similar reductions are also observed in other NV center experiments on both encapsulated and unencapsulated 2D magnets $[19,21]$ and may be due to degradation of the surface layers during sample transfer and laser irradiation, incomplete polarization due to strongly pinned domains, or to submicron scale, stacking-dependent antiferromagnetic interlayer coupling in few-layer samples $[18,19,36]$.

By parking the optical spot outside the flake, we demonstrate sensitive characterization of magnetic hysteresis in ultrathin $\mathrm{CrBr}_{3}$ [8] (see Fig. 9 for data on additional flakes). Figure 2(c) displays the stray field $B_{\mathrm{dc}}^{S}$ due to Flake B as the applied field $B_{\mathrm{dc}}^{E}$ is swept, sensing the magnetization of a local region of the flake near the illuminated $\mathrm{NV}$ location. Lowering $B_{\mathrm{dc}}^{E}$ from $76 \mathrm{mT}$, we observe that the magnetization begins to reverse at $20 \mathrm{mT}$ through the nucleation of domains. The magnetization decreases linearly with field and crosses zero magnetization slowly, revealing that a highly fragmented domain structure is stable and that domain wall pinning impedes the expansion of the reversed domains. Discrete Barkhausen jumps (gray arrows) are observed, corresponding to the hopping of individual domain walls between different pinning sites $[17,20]$. Interestingly, in contrast to the "soft" magnetic behavior of Flake B (nine layers), the hysteresis curve
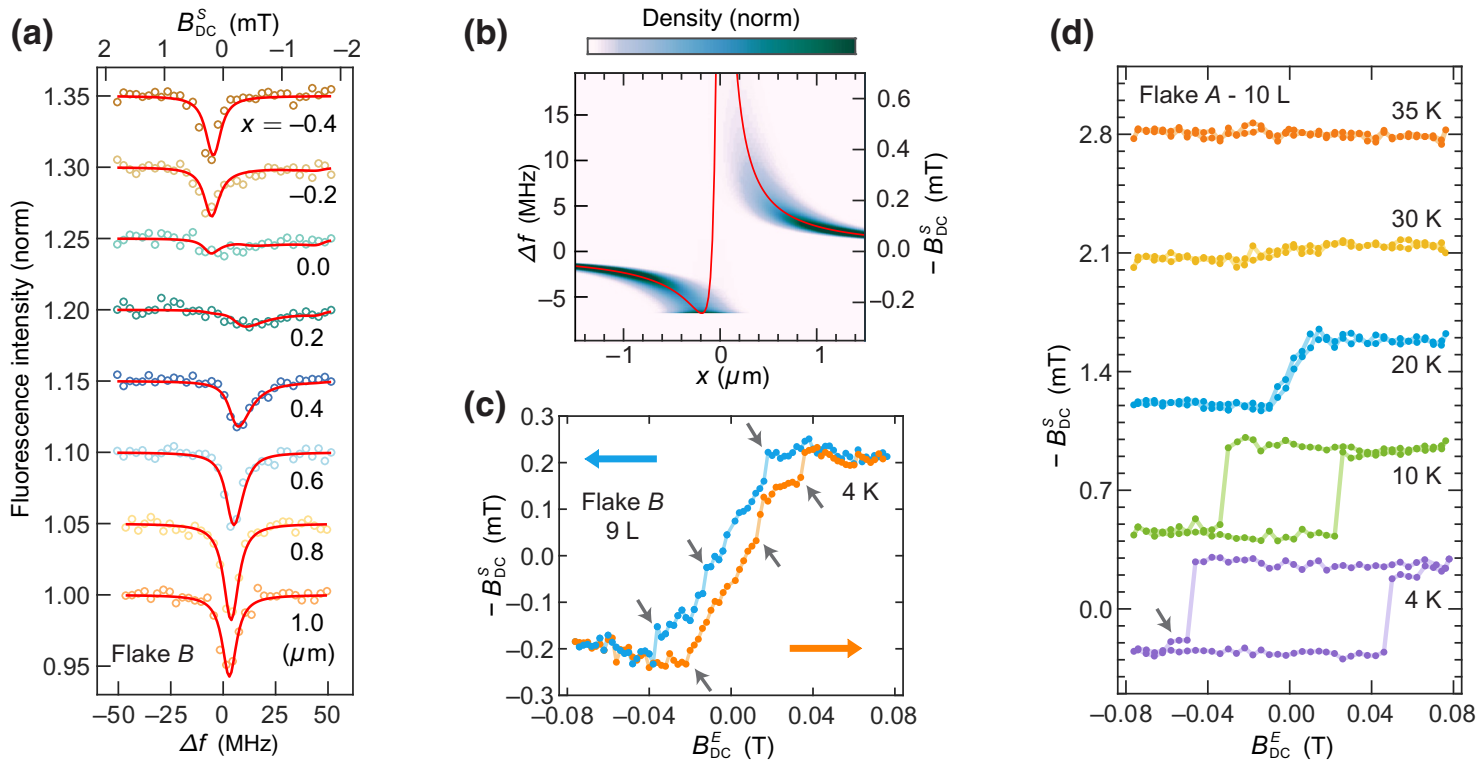

FIG. 2. de magnetometry of ultrathin $\mathrm{CrBr}_{3}$. (a) Linecut of the ODMR spectra across the right edge of Flake B (nine layers) at $B_{\mathrm{dc}}^{E}=76 \mathrm{mT}$ and $T=4 \mathrm{~K}$. Positive (negative) $x$ denotes the exterior (interior) of the flake. Here $\Delta f$ is the shift in frequency from the resonance of NV centers far from the flake $(x \approx 4 \mu \mathrm{m})$. The red lines are fits using a model that considers magnetic field gradients over the optical spot for an ensemble NV sample. (b) Illustration of the distribution of stray fields $B_{\mathrm{dc}}^{S}$ over the optical spot sensed by the NV ensemble. The stray field distribution (density plot) at position $x$ corresponds to the stray field from an infinite magnetized edge (red line) weighted by a Gaussian distribution centered around $x$ for the optical intensity. (c) Magnetic hysteresis curve for Flake B. The magnetization $M$, which is proportional to $-B_{\mathrm{dc}}^{S}$, slowly reverses due to domain wall pinning. Discrete Barkhausen jumps are marked by the arrows. (d) Temperature-dependent hysteresis curves for Flake A (ten layers). Flake A displays rectangular hysteresis, indicating minimal pinning of domain walls once nucleated. 
for a similarly thick flake, Flake A (ten layers), is rectangular, displaying full remanence and a large coercive field [Fig. 2(d)]. Only a single magnetization plateau is observed between the two saturated states, indicating a propensity to form large domains and few pinning centers in the vicinity of the NV center probe. These observations indicate that the metastable domain structures and magnetization reversal process in intermediate thickness $\mathrm{CrBr}_{3}$ flakes (9-10 layers) are extremely sensitive to details of the local microstructure, including strain and defects. As the temperature increases, the hysteresis loop for Flake A narrows, and the magnetization disappears above $30 \mathrm{~K}$.

\section{QUANTUM-ENABLED ac SUSCEPTOMETRY TECHNIQUE}

We now introduce the main innovation of our work: ac susceptometry via spin precession-based ac field sensing. Our protocol builds upon dynamical decoupling (DD) sequences $(\mathrm{XY} 8-N)$ that consist of repeated spin inversions ( $\pi$ pulses) on a NV superposition state undergoing
Larmor precession [37]. Similar to lock-in detection, the DD sequence allows only ac fields synchronized to the $\pi$ pulse spacing to affect the NV precession, while blocking the influence of wideband magnetic noise. This approach extends the coherence time $\left(T_{2}\right)$ of the NV superposition state and significantly enhances the magnetic field sensitivity compared to dc (ODMR) measurements, enabling detection of subtle phenomena in proximal systems, such as nuclear magnetic resonance $[27,38,39]$, electron paramagnetic resonance [40], and photocurrent transport [28]. However, application of DD sequences to ac susceptibility measurements has not been previously achieved, owing to the challenge of extricating a tiny sample response from a much larger ac excitation field.

As outlined in Fig. 3(a), an oscillating coil field with amplitude $B_{\mathrm{ac}}^{C}$ along the NV center axis and frequency $f_{\text {ac }}$ (approximately $200 \mathrm{kHz}$ ) induces an ac magnetization response $\Delta M$ in the sample, which produces its own ac stray field $B_{\mathrm{ac}}^{S}$. After an applied DD sequence with pulse spacing $\tau=1 /\left(2 f_{\text {ac }}\right)$, the NV center precesses by a cumulative angle $\phi_{\mathrm{NV}}$ due to the total resonant ac field parallel (a)

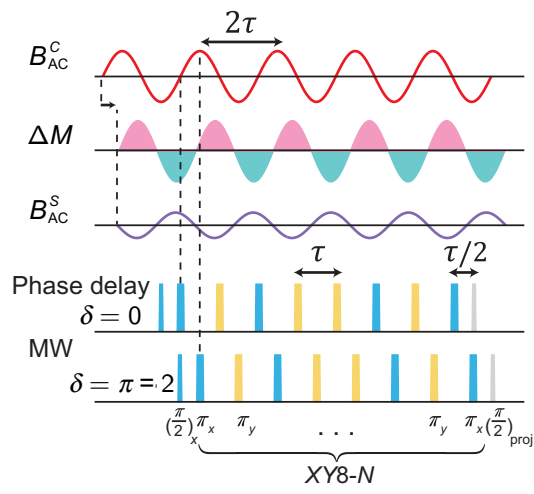

(d)

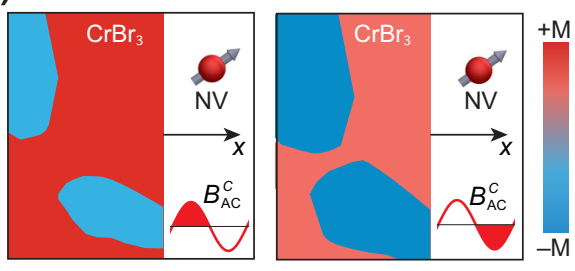

(b)

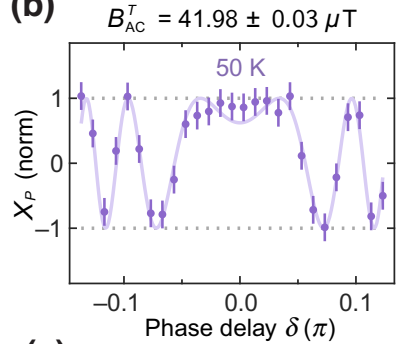

(e)

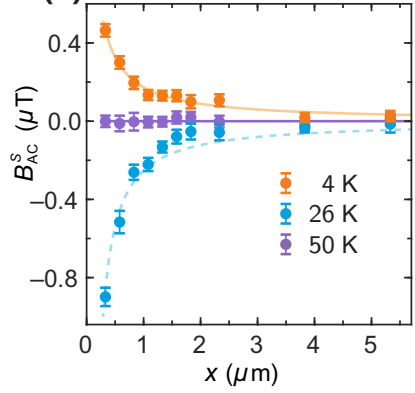

(c)

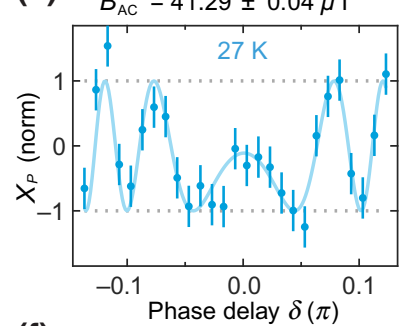

(f)

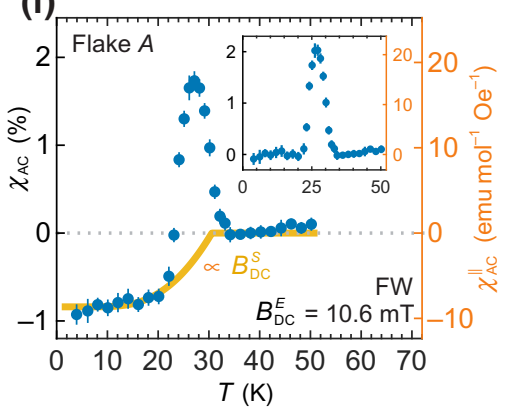

FIG. 3. ac susceptometry technique. (a) Experimental sequence: an oscillating coil field $\vec{B}_{\text {ac }}^{C}$ excites an ac magnetization change $\Delta M$ (with a possible phase shift) in the $\mathrm{CrBr}_{3}$ flake. An XY8- $N$ dynamical decoupling sequence is applied to the NV center to lock-in to the total ac field $B_{\mathrm{ac}}^{T}=B_{\mathrm{ac}}^{C}+B_{\mathrm{ac}}^{S}$ along the NV axis due to both the coil and the sample. The phase delay $\delta$ between $\vec{B}_{\mathrm{ac}}^{C}$ and the microwave (MW) pulses can be tuned to isolate either the in-phase $(\delta=0)$ or out-of-phase $(\delta=\pi / 2)$ magnetization response. (b) The field $B_{\mathrm{ac}}^{T}$ at $50 \mathrm{~K}$ for Flake A determined from the fringe pattern around $\delta=0$ in the $x$ projection, $X_{P}$, of the NV superposition state after precession due to the ac fields. The data are acquired at $B_{\mathrm{dc}}^{E}=10.6 \mathrm{mT}$ using an XY8-N2 sequence with $f_{\mathrm{ac}}=238 \mathrm{kHz}$. (c) The field $B_{\mathrm{ac}}^{T}$ at $27 \mathrm{~K}$. (d) Schematic showing the microscopic mechanisms for $\Delta M$. The field $\vec{B}_{\mathrm{ac}}^{C}$ causes domain wall displacements or magnetization rotation in the sample. (e) Linecut of the sample's ac stray field $B_{\mathrm{ac}}^{S}\left(=B_{\mathrm{ac}}^{T}-B_{\mathrm{ac}}^{C}\right)$ away from Flake A's right edge. A linear background for the coil field $B_{\mathrm{ac}}^{C}$ is determined from the $50 \mathrm{~K}$ data and is subtracted from all three data sets. The positive $B_{\mathrm{ac}}^{S}$ at $4 \mathrm{~K}$ is an artifact induced by the flake's dc stray field $\vec{B}_{\mathrm{dc}}^{S}$. (f) Main: temperature dependence of the uncorrected ac susceptibility $\chi_{\mathrm{ac}}$ for Flake A during field warming (FW) from a saturated state. The left $y$ axis, $\chi_{\mathrm{ac}}$, is defined as $-B_{\mathrm{ac}}^{S} / B_{\mathrm{ac}}^{C}$, while the right $y$ axis, $\chi_{\mathrm{ac}}^{\|}$, is a quantitative estimate for the ac susceptibility parallel to the $c$ axis. The yellow curve represents the baseline for the susceptibility and is proportional to $B_{\mathrm{dc}}^{S}$. Inset: baseline-corrected $\chi_{\mathrm{ac}}$ due to the physical ac magnetization response. 
to the NV axis, $B_{\mathrm{ac}}^{T}=B_{\mathrm{ac}}^{C}+B_{\mathrm{ac}}^{S}$, containing contributions from both the coil and the sample.

The ac susceptibility $\chi_{\mathrm{ac}}=\mu_{0} d M / d B_{\mathrm{ac}}^{C}$, where $\mu_{0}$ is the vacuum permeability, is in general complex valued, with a real (imaginary) part from the in-phase (out-of-phase) sample response. Our DD sequence is phase sensitive by controlling the relative timing, denoted as the phase delay $\delta$, between the applied coil field and the train of $\pi$ pulses applied to the NV center. For $\delta=0$, the NV precession detects $B_{\mathrm{ac}}^{C}+\operatorname{Re}\left(B_{\mathrm{ac}}^{S}\right)$, while for $\delta=\pi / 2$, it detects $\operatorname{Im}\left(B_{\text {ac }}^{S}\right)$ [Fig. 3(a)]. Our measurement scans $\delta$ about $\delta=0$ to map a fringe pattern in the $x$ projection, $X_{P}=\cos \left(\phi_{\mathrm{NV}}\right)$, of the final $\mathrm{NV}$ superposition state on the Bloch sphere. Fitting this fringe pattern allows us to robustly determine $B_{\mathrm{ac}}^{T}$ and resolve $2 \pi$ ambiguities in the NV center's acquired phase (see Appendix D for details). For the small amplitude excitation fields applied, we do not detect any out-of-phase response in multilayer $\mathrm{CrBr}_{3}$ flakes. Hence, $\chi_{\mathrm{ac}}$ in our paper denotes solely the real part of the susceptibility, reflecting reversible magnetization changes. The absence of an imaginary component, due to dissipative processes, is consistent with ac magneto-optic Kerr effect (ac MOKE)-measurements on multilayer $\mathrm{CrBr}_{3}$ flakes at much lower $f_{\mathrm{ac}}=36 \mathrm{~Hz}$, although an imaginary component was observed for monolayer $\mathrm{CrBr}_{3}$ [7].

We first calibrate our measurement by detecting $B_{\mathrm{ac}}^{T}$ near the right edge of Flake $A$ at $B_{\mathrm{dc}}^{E}=10.6 \mathrm{mT}$ and $T=50 \mathrm{~K}$ [Fig. 3(b)]. Far above $T_{c}, B_{\mathrm{ac}}^{T}$ is contributed only by the coil, which we determine to produce a peak amplitude of $41.98 \pm 0.03 \mu \mathrm{T}$ along the NV center axis. Over the 33.6 $\mu$ s duration of the XY8-N2 sequence used here, the coil field induces a large maximum precession angle $\phi_{\mathrm{NV}} \approx$ $50 \pi$ for $\delta=0$ and leads to rapid oscillations in $X_{P}$ for small changes in $\delta$. Tracking the same spot relative to the flake's edge, we find that $B_{\mathrm{ac}}^{T}$ is reduced to $41.29 \pm 0.04 \mu \mathrm{T}$ at $27 \mathrm{~K}$ [Fig. 3(c)]. We verify that the coil field $B_{\mathrm{ac}}^{C}$ does not change with temperature by measuring $\mathrm{NV}$ centers far from any flakes (see Fig. 12). Hence, we conclude that Flake A produces an ac stray field $B_{\mathrm{ac}}^{S} \approx-0.69 \mu \mathrm{T}$, which is $>300$ times smaller than its de stray field at saturation.

As illustrated Fig. 3(d), the susceptibility $\chi_{\mathrm{ac}}$ is contributed microscopically by both magnetization rotation (MR) and domain wall displacement (DWD). For MR, the coil field $\vec{B}_{\text {ac }}^{C}$ exerts a torque that aligns sample spins with variable orientations along the direction of $\vec{B}_{\mathrm{ac}}^{C}$, predominantly along the $z$ axis in our experiment, also the easy axis for $\mathrm{CrBr}_{3}$. Alternatively, for DWD, domains aligned with $\vec{B}_{\text {ac }}^{C}$ grow in area at the expense of antialigned domains. Hence, for both MR and DWD, $\Delta M$ of the flake is positively correlated to $\vec{B}_{\mathrm{ac}}^{C}$. This implies that the sample field $B_{\mathrm{ac}}^{S}$ along the NV center axis should indeed have opposite sign to the coil field $B_{\mathrm{ac}}^{C}$ outside the flake's right edge [cf. Fig. 1(c)].

Mapping $B_{\mathrm{ac}}^{S}$ versus $x$ in Fig. 3(e), we corroborate that Flake A's ac stray field is negative at $26 \mathrm{~K}$ and decays away from the flake's edge; however, a surprising positive signal is observed at $4 \mathrm{~K}$. The linecuts in Fig. 3(e) are obtained during $\mathrm{FW}$ at $B_{\mathrm{dc}}^{E}=10.6 \mathrm{mT}$ after polarizing the flake with $B_{\mathrm{dc}}^{E}=76 \mathrm{mT}$. In this case, $\chi_{\mathrm{ac}}$ should be zero starting in the saturated state at $4 \mathrm{~K}\left[d M / d B_{\mathrm{dc}}^{E}=0\right.$ in Fig. 2(d)]. The observed positive signal is a measurement artifact that is induced by the flake's temperature-dependent dc stray field $\vec{B}_{\mathrm{dc}}^{S}$. As the flake is approached, the total dc field $\vec{B}_{\mathrm{dc}}^{E}+\vec{B}_{\mathrm{dc}}^{S}$ becomes misaligned from the NV center axis, and the NV spin precession is affected not only by $B_{\mathrm{ac}}^{C}$ (parallel to the NV axis), but also to a lesser degree by the component of coil field perpendicular to the NV axis. Theoretical analysis shows that the contribution to $\phi_{\mathrm{NV}}$ due to the perpendicular component of the coil field depends linearly on the dc field perpendicular to the NV axis for small fields (see Appendix E). This approximately $1 \%$ systematic deviation is resolved by the high precision and stability of our measurements.

Accordingly, the spatial dependence of the $\operatorname{artifact} B_{\mathrm{ac}}^{S}$ at $4 \mathrm{~K}$ fits accurately to the decay (orange line) of the dc stray field perpendicular to the NV center axis due to the edge of a homogeneous, out-of-plane magnetized sheet [35]. On the other hand, the spatial dependence $B_{\mathrm{ac}}^{S}$ at $26 \mathrm{~K}$, containing physical signals from ac magnetization changes inside the flake, displays a faster decay that is better fit by the stray field parallel to the NV axis from the same magnetic edge (dashed blue line).

To extract the physical ac susceptibility signal, we present the full temperature dependence of $B_{\mathrm{ac}}^{S}$ in Fig. 3(f). The data here are measured at fixed location $(x \approx 0.33 \mu \mathrm{m})$ during field-warming Flake A from the saturated state. We define a subjective metric, $\chi_{\mathrm{ac}} \equiv-B_{\mathrm{ac}}^{S} / B_{\mathrm{ac}}^{C}$, where $B_{\mathrm{ac}}^{S}$ depends on the measurement location. Since the artificial contribution to $B_{\mathrm{ac}}^{S}$ is proportional to the dc field perpendicular to the $\mathrm{NV}$ axis, we should subtract a baseline proportional to the temperature-dependent dc magnetization of the flake. For each temperature during the sweep, we simultaneously sample the dc magnetization by measuring $B_{\mathrm{dc}}^{S}$ via ODMR. The baseline for $\chi_{\mathrm{ac}}$ [yellow curve in Fig. 3(f)] is thus determined by scaling the fitted $B_{\mathrm{dc}}^{S}$ data by a multiplicative factor to match the uncorrected $\chi_{\mathrm{ac}}$ data for $T<10 \mathrm{~K}$, where the physical susceptibility should be zero (see Fig. 11 for raw data points and a fitted baseline).

The baseline-subtracted $\chi_{\mathrm{ac}}$, displayed in the inset of Fig. 3(f), reveals the onset of susceptibility via domain nucleation upon warming to $T=20 \mathrm{~K}$. The susceptibility $\chi_{\text {ac }}$ peaks at $27 \mathrm{~K}$, below $T_{c}=30.5 \pm 0.5 \mathrm{~K}$ of Flake A [determined by field cooling (FC) initial susceptibility measurements presented in the next section]. A peak in $\chi_{\mathrm{ac}}$ below $T_{c}$ is commonly observed in ferromagnets and is known generically as a Hopkinson peak [23,41]. The Hopkinson peak for the FW trace here originates from an increase in domain wall density and mobility as the temperature increases, which is ultimately counterbalanced by the loss of saturation magnetization $M_{S}$ towards $T_{c}$. 
An advantage of NV magnetometry is its potential for quantitative, material-independent characterization of $\chi_{\mathrm{ac}}$, since the phase precession $\phi_{\mathrm{NV}}$ is governed solely by the gyromagnetic ratio $\gamma_{e}$ and experimental parameters. This is in contrast to the Kerr angle in ac MOKE, which depends on the specific material's spin-resolved band structure [16] and can vanish in certain materials, such as some antiferromagnets, that lack appropriate symmetry [42]. Microscopically, the ac susceptibility can vary spatially, and the conventional notion of a single value for susceptibility averages over these variations.

Determining the spatial distribution of the ac magnetization $\Delta M(\vec{r})$ from the stray field requires full twodimensional imaging and some additional constraint [43]. Nevertheless, a local measurement of $B_{\mathrm{ac}}^{S}$ outside the flake permits a quantitative estimate for the spatially averaged magnetization response $\overline{\Delta M}$ that becomes accurate when the source of the susceptibility is spatially dense. The relevant length scale is the distance $x$ between the NV probe location and the flake's edge. An accurate estimate applies, for example, when the ac susceptibility is contributed by paramagnetic spin rotation, which is nearly homogeneous, or by domain walls with separations that are sufficiently small compared to $x$. In this case, the stray field $B_{\mathrm{ac}}^{S}$ produced outside the flake by $\Delta M(\vec{r})$ approaches that produced by a homogeneous change in the flake's magnetization by $\overline{\Delta M}$. To illustrate, our simulations show that $B_{\mathrm{ac}}^{S}$ at $x=0.33 \mu \mathrm{m}$ produced by DWDs of stripe domains with $100 \mathrm{~nm}$ width differs by only about $2 \%$ from the field produced by distributing the total magnetization change uniformly over the flake (see Appendix F). Thus, we can determine the spatially averaged ac susceptibility $\chi_{\mathrm{ac}}^{\|}$for fields parallel to the easy axis of $\mathrm{CrBr}_{3}$ by computing the uniformly distributed $\overline{\Delta M}$ that produces the same $B_{\mathrm{ac}}^{S}$ measured at the NV location. The resulting $\chi_{\mathrm{ac}}^{\|}$ in emu $\mathrm{mol}^{-1} \mathrm{Oe}^{-1}$ [left $y$ axis in Fig. 3(f)] accounts for experimental parameters such as the angle and distance to the flake's edge and provides a quantitative measure that can be compared between different flakes and other experiments.

\section{INITIAL ac SUSCEPTIBILITY}

Having detailed our experimental technique and analysis, we now present baseline-corrected measurements of $\chi_{\mathrm{ac}}$ for few-layer $\mathrm{CrBr}_{3}$ during the initial magnetization process from the paramagnetic state. We begin with Flake $\mathrm{C}$, a thicker 18-layer flake, that displays a hysteresis loop with minimal opening area at $4 \mathrm{~K}$ (see Fig. 9), approaching the linear retraceability observed for bulk samples [32]. As shown in Fig. 4(a) (red points), the dc stray field $B_{\mathrm{dc}}^{S}$ for Flake $C$ remains virtually vanishing throughout $\mathrm{FC}$ at small $B_{\mathrm{dc}}^{E}=2 \mathrm{mT}$, indicating the stabilization of an equally balanced, fine-grained domain structure. Electron microscopy observes periodic stripelike domains in bulk $\mathrm{CrBr}_{3}$ with widths of approximately $100 \mathrm{~nm}$ [44], consistent with significantly reduced stray fields even when measured at our near-field location. The domains, however, can be polarized predominantly along the dc field at $B_{\mathrm{dc}}^{E}=76 \mathrm{mT}$. During subsequent FW, the magnetization, proportional to $-B_{\mathrm{dc}}^{S}$ (purple points), is well described by a critical model $M(T) \propto\left(1-T / T_{c}\right)^{\beta}$ with $\beta=0.38 \pm 0.02$ and $T_{c}=30.5 \mathrm{~K}$. These results are in close agreement with
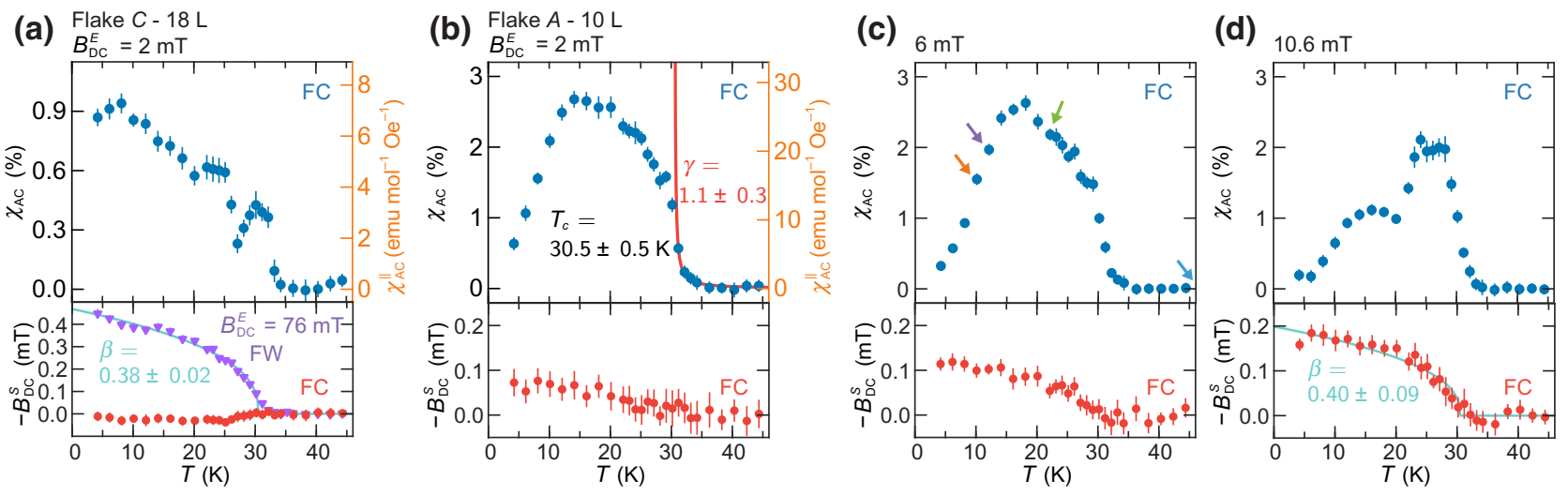

FIG. 4. Initial ac susceptibility for bulklike and ultrathin $\mathrm{CrBr}_{3}$. (a) $\chi_{\mathrm{ac}}$ (blue) for bulklike Flake $\mathrm{C}$ (18 layers) during field cooling (FC) at low bias field $B_{\mathrm{dc}}^{E}=2 \mathrm{mT}$. The dc magnetization (red), proportional to $-B_{\mathrm{dc}}^{S}$, remains zero during FC, indicating a finely fragmented domain structure. The magnetization during $\mathrm{FW}$ (purple) at a polarizing field $B_{\mathrm{dc}}^{E}=76 \mathrm{mT}$ follows a critical scaling $M(T) \propto\left(1-T / T_{c}\right)^{\beta}$ with $\beta=0.38 \pm 0.02$. (b)-(d) The susceptibility $\chi_{\mathrm{ac}}$ (blue) and $B_{\mathrm{dc}}^{S}$ (red) for Flake A (10 layers) during FC at (b) $B_{\mathrm{dc}}^{E}=2 \mathrm{mT}$, (c) $6 \mathrm{mT}$, and (d) $10.6 \mathrm{mT}$. Flake A displays a rectangular hysteresis curve more common in ultrathin $\mathrm{CrBr}_{3}$. The quantitative susceptibility, $\chi_{\mathrm{ac}}^{\|}$, is larger for Flake A than Flake $\mathrm{C}$ at $B_{\mathrm{dc}}^{E}=2 \mathrm{mT}$. The susceptibility $\chi_{\mathrm{ac}}$ diverges at $T_{c}=30.5 \mathrm{~K}$ with a critical exponent $\gamma=1.1 \pm 0.3$ [red fit in (b), upper], while the dc magnetization scales with a critical exponent $\beta=0.40 \pm 0.09$ [cyan fit in (d), lower]. The arrows in (c) indicate temperatures for frequency-dependent measurements presented in Fig. 5 . All $\chi_{\text {ac }}$ data here are taken at $f_{\mathrm{ac}}=238 \mathrm{kHz}$ with XY8- 2 and are baseline corrected using the flake's dc stray field $B_{\mathrm{dc}}^{S}$. 
prior works that found $\beta \approx 0.4$ for both bulk [45] and monolayer $\mathrm{CrBr}_{3}$ [34].

In contrast to its vanishing de magnetization, the ac susceptibility $\chi_{\mathrm{ac}}$ for Flake $\mathrm{C}$ reveals distinct features that highlight the formation and mobility of domains [Fig. 4(a), blue points]. As $T_{c}$ is approached, the paramagnetic divergence of $\chi_{\mathrm{ac}}$ is stunted, and $\chi_{\mathrm{ac}}$ decreases initially, leading to a local maximum slightly below $T_{c}$ (Hopkinson peak). Monte Carlo simulations [46,47] indicate that incipient domainlike patches begin to form a few kelvin above $T_{c}$, with the magnetic axes of the domains initially isotropically distributed. We attribute the initial rounding and decrease of $\chi_{\mathrm{ac}}$ to the formation of this domain structure, which impedes coherent MR relative to the paramagnetic state.

As temperature is reduced further, the measured $\chi_{\mathrm{ac}}$ for Flake C starts to steadily increase [Fig. 4(a)]. Because of increasing magnetic anisotropy, we expect a crossover to a $180^{\circ}$ domain phase with magnetizations fully parallel or antiparallel to the easy $(z)$ axis $[46,47]$. Hence, $\chi_{\mathrm{ac}}$ becomes dominated by DWD, while the MR contribution is phased out as the magnetization becomes mostly parallel to $\vec{B}_{\text {ac }}^{C}$. For DWDs described by the membranelike bulging of a domain wall between two pinned endpoints, the susceptibility $\chi_{\mathrm{DW}}$ is proportional to $M_{S}^{2} l / \gamma_{\mathrm{DW}}$, where $M_{S}$ is the saturation magnetization, $l$ is the length between the pinned ends, and $\gamma_{\mathrm{DW}} \propto \sqrt{A_{\mathrm{ex}} K}$ is the domain wall energy [26]. Here, $A_{\text {ex }}$ is the exchange stiffness parameter and $K$ is the uniaxial anisotropy.

Making the approximations $A_{\mathrm{ex}} \propto M_{S}^{2}$ and $K \propto M_{S}^{3}$ for bulk $\mathrm{CrBr}_{3}$ [48], the factor $M_{S}^{2} / \gamma_{\mathrm{DW}}\left(\propto M_{S}^{-1 / 2}\right)$ would decrease with decreasing temperature. Thus, the increasing $\chi_{\mathrm{ac}}$ observed experimentally instead suggests that the length $l$ of the domain wall that can be continuously displaced is increasing for a range of decreasing temperatures, assuming constant domain wall density [44]. This could reflect the change in the domain morphology observed in imaging experiments on bulk $\mathrm{CrBr}_{3}$, where smoother, straighter striped domains evolve at low temperature from zigzag stripes near $T_{c}$ with short interlocking sections [44,49]. Quantitatively, the peak value of $\chi_{\mathrm{ac}}^{\|}$ [approximately $7 \mathrm{emu} /(\mathrm{mol} \mathrm{Oe})$ ] determined for Flake C by NV magnetometry is close to that measured for bulk $\mathrm{CrI}_{3}$ using a SQUID magnetometer [approximately $6 \mathrm{emu} /$ (mol Oe)] [50].

We next present $\chi_{\mathrm{ac}}$ measurements for Flake A, which instead displays a rectangular hysteresis curve at $4 \mathrm{~K}$ [Fig. 2(d)] more characteristic of ultrathin flakes (e.g., monolayer). For FC at low $B_{\mathrm{dc}}^{E}=2 \mathrm{mT}$ [Fig. 4(b)], we see a stronger divergence of the paramagnetic susceptibility in quantitative units for Flake A (3 times larger than Flake C), suggesting reduced interference from its domain structure. This allows us to extract $T_{c}=30.5 \pm 0.5$ $\mathrm{K}$ from the inflection point in $\chi_{\mathrm{ac}}$ versus temperature and a critical exponent $\gamma=1.1 \pm 0.3$ in the scaling of $\chi_{\mathrm{ac}}(T) \propto$ $\left(T / T_{c}-1\right)^{\gamma}$ above $T_{c}$. This value of $\gamma$ signals that Flake A is still proximal to the bulk, mean-field limit of magnetic interactions $(\gamma=1)$ [45]. However, its domain dynamics below $T_{c}$, as revealed through ac susceptibility, differs from the thicker Flake C.

For Flake A, we observe only a small kink in $\chi_{\text {ac }}$ at $29 \mathrm{~K}$, rather than the broad Hopkinson peak seen for Flake C. The susceptibility $\chi_{\mathrm{ac}}$ reaches a maximum at an intermediate temperature (approximately $14 \mathrm{~K}$ ), before decreasing to a small value at $4 \mathrm{~K}$. The high peak value of $\chi_{\mathrm{ac}}$ is consistent with the reduced density of pinning sites suggested by the rectangular hysteresis curve. The eventual decrease of $\chi_{\mathrm{ac}}$ at lower temperatures reflects the combination of two factors. First, the net area of domain walls near the NV location decreases as the magnetic domains antiparallel to $B_{\mathrm{dc}}^{E}$ shrink or disappear to minimize the domain wall energy [46]. This process is substantiated by the increase in the dc magnetization of the flake during FC [Fig. 4(b), red data]. Second, the mobility for the remaining domain walls is reduced due to increasing magnetic anisotropy $K$ at lower temperatures, which will be corroborated by frequency-dependent measurements.

Our measurements suggest that differing domain morphologies arise during the thermal magnetization of bulk versus few-layer $\mathrm{CrBr}_{3}$. For Flake $\mathrm{C}$, the absence of a dc stray field and the low absolute value of $\chi_{\mathrm{ac}}$ throughout field cooling indicate the formation of nanoscale domains with low mobility, while for Flake A, more mobile domain walls (high $\chi_{\mathrm{ac}}$ ) consolidate into larger domains, leading to an increasing dc stray field with cooling. Our observations may be associated with imaging experiments that visualized disordered, patchlike domains in three- and four-layer $\mathrm{CrBr}_{3}$ [20] and narrow stripe domains in bulk $\mathrm{CrBr}_{3}$ [44].

To gain further insight, we examine how features in the temperature dependence of $\chi_{\mathrm{ac}}$ for Flake A evolve with increasing bias field $B_{\mathrm{dc}}^{E}$ during FC. As shown in Figs. 4(b)-4(d), the behavior of $\chi_{\mathrm{ac}}$ for different $B_{\mathrm{dc}}^{E}$ closely overlaps in the paramagnetic region, but separates below $T_{c}$. For larger $B_{\mathrm{dc}}^{E}$, the enhanced field pressure $p=2 M_{S} B_{\mathrm{dc}}^{E}$ accelerates the depinning and expansion of domains aligned with the field, causing a more rapid rise in the dc magnetization. Concomitantly, $\chi_{\text {ac }}$ peaks at higher temperatures during $\mathrm{FC}$ and terminates at lower values at $4 \mathrm{~K}$, reflecting accelerated reduction of domain wall area.

These effects are accentuated for FC at the highest $B_{\mathrm{dc}}^{E}=$ $10.6 \mathrm{mT}$ [Fig. 4(d)], where $\chi_{\mathrm{ac}}$ rapidly decreases, while $B_{\mathrm{dc}}^{S}$ increases below $T_{c}$, signaling a more homogeneous magnetic state. The unusual dual-humped shape of $\chi_{\mathrm{ac}}$ during FC [Fig. 4(d)] is not reproducible during FW at the same $B_{\mathrm{dc}}^{E}$ [Fig. 3(f)]. Hence, its mechanism should relate to the hysteretic nature of domains, rather than to an intrinsic transition. Since the dc magnetization approaches the saturated, single-domain value and $\chi_{\mathrm{ac}}$ drops to nearly zero 
by the end of FC, we are probing the motion of only a few domain walls in the vicinity of the NV center. A possible explanation for the turnaround of $\chi_{\mathrm{ac}}$ at $20 \mathrm{~K}$ could thus be the thermal depinning of a single domain wall from a strong pinning center, analogous to a Barkhausen jump in the dc hysteresis measurement. Discontinuous ac susceptibility of single domain walls has been reported by micro-Hall magnetometry that similarly probed a mesoscopic sample area [51]. Alternatively, for FC at larger $B_{\mathrm{dc}}^{E}$, the flake may first form a homogeneous magnetic state below $T_{c}$, tilted in the direction of $\vec{B}_{\mathrm{dc}}^{E}\left(54.7^{\circ}\right.$ to the easy axis), and only nucleate magnetic domains at lower temperatures. The dual peaks in $\chi_{\mathrm{ac}}$ could then correspond to the transition between the MR and DWD contributions to $\chi_{\mathrm{ac}}$. Future work incorporating dc imaging could clarify the relationship between specific domain configurations and features in local $\chi_{\mathrm{ac}}$ measurements.

\section{FREQUENCY-DEPENDENT ac SUSCEPTIBILITY}

Finally, we demonstrate frequency-dependent ac susceptibility measurements by sweeping $f_{\text {ac }}$ of the coil field, while changing the repetition rate of the NV $\pi$ pulses to maintain the lock-in condition. We increase the number of $\pi$ pulses for larger $f_{\text {ac }}$ so that the total duration of the sensing sequence remains roughly constant, giving us similar precision on the determination of $\chi_{\mathrm{ac}}$. For $B_{\mathrm{dc}}^{E}=6 \mathrm{mT}$, we gather data for $f_{\text {ac }}$ spanning $119 \mathrm{kHz}$ (using $8 \pi$ pulses) to $714 \mathrm{kHz}$ (48 $\pi$ pulses). The lower range is limited by the ${ }^{13} \mathrm{C}$ nuclear spin bath inside diamond, which reduces the $\mathrm{NV}$ center coherence as $f_{\text {ac }}$ overlaps the ${ }^{13} \mathrm{C}$ Larmor frequency $(64 \mathrm{kHz})$, and the upper by the finite duration of our $\pi$ pulse. Intrinsically, NV susceptometry is applicable to a wider frequency range, leveraging longer coherence times in surface-engineered, isotopically purified samples [52] and faster spin inversions in the strong driving regime (> $100 \mathrm{MHz}$ effective Rabi frequency [53]).

As displayed in Fig. 5, $\chi_{\mathrm{ac}}$ for Flake A is independent of $f_{\mathrm{ac}}$ well above $T_{c}(50 \mathrm{~K}$, blue), as expected. At $22 \mathrm{~K}$, however, during the initial rise of the field-cooling $\chi_{\mathrm{ac}}$ [green arrow in Fig. 4(c)], we observe a weak decrease in $\chi_{\mathrm{ac}}$ for higher excitation frequencies. This effect becomes more pronounced upon further cooling, as $\chi_{\mathrm{ac}}$ begins to decrease with temperature. Increasing $f_{\text {ac }}$ sixfold from 119 to $714 \mathrm{kHz}$, the susceptibility $\chi_{\mathrm{ac}}$ is reduced by a factor of $0.90 \pm 0.08$ at $22 \mathrm{~K}, 0.78 \pm 0.05$ at $12 \mathrm{~K}$, and $0.68 \pm 0.10$ at $10 \mathrm{~K}$.

As first elucidated by Döring [54], the displacement $s$ of a domain wall satisfies the phenomenological equation

$$
m d^{2} s / d t^{2}+\beta d s / d t+\alpha s=2 M_{S} H
$$

where $m$ is the apparent mass of the wall, $\beta$ is the viscous damping, $\alpha$ is the restoring force due to the local potential,

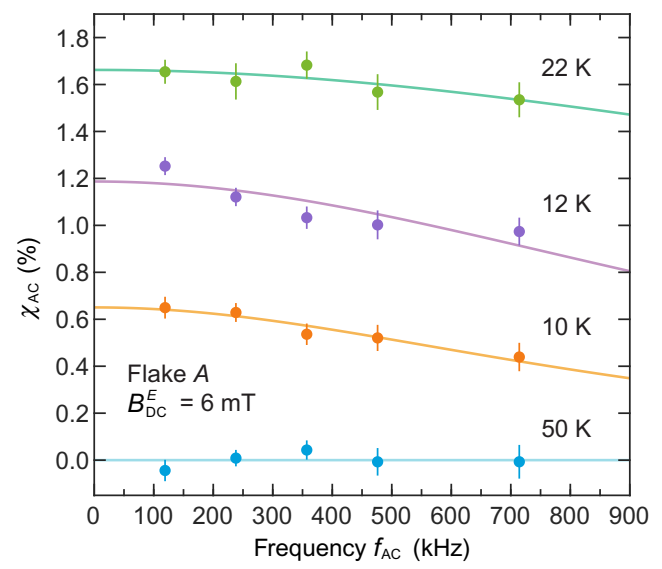

FIG. 5. Frequency-dependent susceptibility of domain walls in ultrathin $\mathrm{CrBr}_{3}$. The susceptibility $\chi_{\mathrm{ac}}$ for Flake $\mathrm{A}$ at $50 \mathrm{~K}$ (blue), $22 \mathrm{~K}$ (green), $12 \mathrm{~K}$ (purple), and $10 \mathrm{~K}$ (orange) during FC. The solid lines are fits using a damped, driven domain wall oscillation model. The number of $\pi$ pulses increases for larger $f_{\text {ac }}$ (e.g., $\mathrm{XY} 8-\mathrm{N} 6$ is used for $714 \mathrm{kHz}$ ).

and $2 M_{S} H$ is the driving force due to the field pressure on a $180^{\circ}$ domain wall. For an oscillating field $H=H_{0} e^{i \omega t}$ with $\omega=2 \pi f_{\text {ac }}$, the susceptibility $\chi_{\mathrm{DW}}$ is proportional to the displacement $s$ and is thus analogous to the transfer function of a driven damped harmonic oscillator:

$$
\chi_{\mathrm{DW}}=\frac{\chi_{0}}{1-\left(\omega / \omega_{0}\right)^{2}+i\left(\omega / \omega_{c}\right)} .
$$

Here $\chi_{0}$ is the dc susceptibility, $\omega_{0}=\sqrt{\alpha / m}$ is the domain wall resonance, and $\omega_{c}=\alpha / \beta$ is a relaxation frequency, reflecting the timescale $\tau_{c}=\beta / \alpha$ for displacements to decay. For dc fields $H$, the phenomenological mobility $\mu=(d s / d t) / H$ of a uniform domain wall is $2 M_{S} / \beta$ in this model [55].

The domain wall resonance $\omega_{0}$ for thin $\mathrm{CrBr}_{3}$ platelets has been measured to exceed $700 \mathrm{MHz}$ [56], and hence $\left(\omega / \omega_{0}\right)^{2}$ can be neglected for excitation frequencies applied here, leaving

$$
\operatorname{Re}\left(\chi_{\mathrm{DW}}\right)=\frac{\chi_{0}}{1+\left(\omega / \omega_{c}\right)^{2}} .
$$

The solid lines in Fig. 5 show fits of our data to Eq. (3). The fitted $\omega_{c} / 2 \pi$ decreases monotonically from approximately $2 \mathrm{MHz}$ at $22 \mathrm{~K}$ to approximately $1 \mathrm{MHz}$ at 10 $\mathrm{K}$, indicating longer relaxation times, resulting most likely from stronger domain wall damping $\beta$. For ferromagnetic insulators, $\beta$ is contributed by spin-lattice relaxation that similarly damps magnetization precession (Gilbert damping) and is proportional to $M_{S} \sqrt{K / A}$, which should indeed increase with decreasing temperature [57]. In context, the robustness of $\chi_{\mathrm{ac}}$ here to frequencies exceeding hundreds of kilohertz contrasts with the behavior of conventional 
ferromagnetic materials that are commonly characterized in commercial SQUID magnetometers with bandwidths of a few kilohertz. Our measurements thus underscore the overall low damping and high mobility of domain walls in ultrathin $\mathrm{CrBr}_{3}$ [46].

\section{CONCLUSION}

The ac susceptometry technique developed here establishes NV magnetometry as a true multimodal probe for ultrathin magnetic materials, combining nanoscale spatial resolution and quantitative magnetization characterization previously demonstrated using dc field measurements [1821] with now access to a wide bandwidth of dynamical magnetization phenomena. Although we do not measure monolayer samples, the precision on our ac field measurements (approximately $40 \mathrm{nT}$ ), enabled by our active protection of the $\mathrm{NV}$ spin coherence, exceeds that of prior dc magnetometry on 2D magnetic monolayers by 2 orders of magnitude. This enhanced sensitivity allows us to resolve the ac magnetic response due to paramagnetic spin rotation and domain wall motion in single, few-layer $\mathrm{CrBr}_{3}$ flakes, showing the latter to be strongly influenced by the domain morphology and local pinning landscape of the flake. A future opportunity lies in the investigation of the out-of-phase component of ac susceptibility, which can be compared through the fluctuation-dissipation theorem to simultaneous spin noise spectra measured by NV magnetometry [58]. Moreover, the technique and analysis introduced here, although utilizing high-fidelity control and longer averaging, are extensible to scanning NV measurements for the nanoscale imaging of ac susceptibility $[18,20,21]$. Our exciting development thus opens the door to understanding subgigahertz magnetic dynamics in diverse 2D materials, including antiferromagnets [13], superconductors [59], and quantum spin liquids [60] that lack magneto-optical coupling, and in single molecule magnets [61] and superparamagnetic nanoparticles [62] at the single particle limit.

\section{ACKNOWLEDGMENTS}

The authors thank B. Flebus and Q. Ma for valuable discussions. B.B.Z acknowledges support from the National Science Foundation under Grant No. ECCS-2041779. F.T. acknowledges support from the National Science Foundation under Grant No. DMR-1708929. M.J.G. is supported by the National Science Foundation under Grant No. DMR-2003343, and K.S.B. is supported by the Office of Naval Research under Grant No. N00014-20-1-2308.

X.-Y.Z. and B.B.Z. devised the experiment. X.-Y.Z. and Y.-X.W. built the cryogenic confocal system. X.-Y.Z. acquired and analyzed the data with assistance from Y.X.W., T.D., and B.B.Z. T.A.T and F.T. synthesized the $\mathrm{CrBr}_{3}$ samples. M.J.G. and K.S.B maintained the glovebox and AFM, and instructed X.-Y.Z. on their use. B.B.Z. and X.-Y.Z. wrote the paper with contributions from all authors.

\section{APPENDIX A: EXPERIMENTAL SETUP}

We optically address ensemble NV centers in a diamond chip placed in a closed-cycle cryostat (Montana Instruments) using a home-built scanning confocal microscope. A $515 \mathrm{~nm}$ diode laser (Cobolt 06-MLD) is focused on NV centers with an objective (Olympus MPlanFL N 100x) for optical initialization and spin readout. The laser power incident on the back of the objective is $40 \mu \mathrm{W}$. PL from the NV center is collected by the same objective. It is then band-pass filtered between $690-800 \mathrm{~nm}$ (Omega 690LP and Thorlabs FESH0800) and recorded by a single-photon counting module (Excelitas). Gigahertz microwaves are driven by a signal generator (SRS SG386) and an amplifier (Mini-Circuits ZHL-16W-43-S+). Kilohertz waveforms for ac excitation of the ferromagnet are driven by a function generator (Siglent SDG2042X). They are joined together by a diplexer (Mini-Circuits ZX752R15-S+) and delivered to a wire loop placed on top of the diamond chip. The wire loop is soldered to a printed circuit board that is mounted onto the same copper sample holder on which the diamond is glued. Measurement protocols including pulse timing and IQ mixing are controlled by an arbitrary waveform generator (Tektronix AWG5014C).

The diamond substrate is treated with ${ }^{15} \mathrm{~N}$ ion implantation at an area dose of $10^{12}$ ions $/ \mathrm{cm}^{2}$ and $45 \mathrm{keV}$ energy to create an ensemble layer at approximately $60 \mathrm{~nm}$ depth. Subsequently, the sample is annealed at $1050^{\circ} \mathrm{C}$ for one hour in argon forming gas to mobilize vacancies and create NV centers. We estimate that approximately $100 \mathrm{NV}$ centers are probed within our diffraction-limited spot size.

Single crystals of $\mathrm{CrBr}_{3}$ are grown using chemical vapor transport by heating a mixture of $\mathrm{Cr}+0.75 \mathrm{TeBr}_{4}$ at $650^{\circ} \mathrm{C}$ for $72 \mathrm{~h}[32,33] . \mathrm{CrBr}_{3}$ thin flakes are exfoliated using Nitto blue tape directly onto the bare diamond surface without encapsulation. The exfoliation is carried out in an argon-filled glovebox in which $\mathrm{O}_{2}$ and $\mathrm{H}_{2} \mathrm{O}$ levels are maintained $<1 \mathrm{ppm}$. The exposure to ambient during sample transfer to our cryostat and before being pumped to $10^{-5}$ mbar is estimated to be less than $15 \mathrm{~min}$.

The $T_{2}$ coherence time of the ensemble sample used is $125 \mu$ s under an XY8- $N 1$ sequence. As shown in Fig. 6, the Rabi contrast of the sample is temperature dependent, which may be due to the intrinsic photodynamics of the NV center at low temperature. Accordingly, a single $\chi_{\mathrm{ac}}$ data point extracted from a full phase delay $(\delta)$ sweep [e.g., Figs. 3(b) and 3(c)] takes about $20 \mathrm{~min}$ at $4 \mathrm{~K}$, but $90 \mathrm{~min}$ at $30 \mathrm{~K}$. With optimal readout contrast at $4 \mathrm{~K}$, we typically use approximately $7 \times 10^{5}$ green laser readouts, split between the $+X_{P}$ and $-X_{P}$ projections (differential measurement), for each value of $\delta$. 


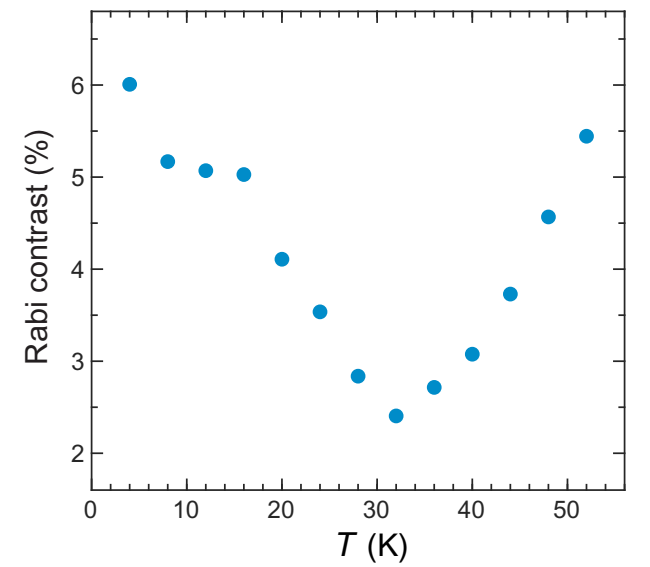

FIG. 6. Rabi contrast versus temperature. The Rabi contrast of the ensemble NV sample used in these experiments reaches a minimum at approximately $30 \mathrm{~K}$.

\section{APPENDIX B: ADDITIONAL CHARACTERIZATION OF $\mathrm{CrBr}_{3}$ FLAKES}

Atomically thin $\mathrm{CrBr}_{3}$ samples are exfoliated directly onto the diamond surface. Their thicknesses are identified with AFM followed by histogramming the height within a region of interest. The thicknesses are summarized in Fig. 7 where a step size of $0.7 \mathrm{~nm}$ and an offset of $0.4 \mathrm{~nm}$ are observed; thus, the layer numbers of different flakes are determined. Figure 8 shows AFM topography of all the samples summarized in Fig. 7. The dc hysteresis curves of the different flakes, measured at $4 \mathrm{~K}$, are presented in Fig. 9, if not already presented in the main text. In Fig. 9(c), we show the $\chi_{\mathrm{ac}}$ (blue) and $B_{\mathrm{dc}}^{S}$ (red) curves during field-cooling Flake D (15 layers) at $B_{\mathrm{dc}}^{E}=2 \mathrm{mT}$.

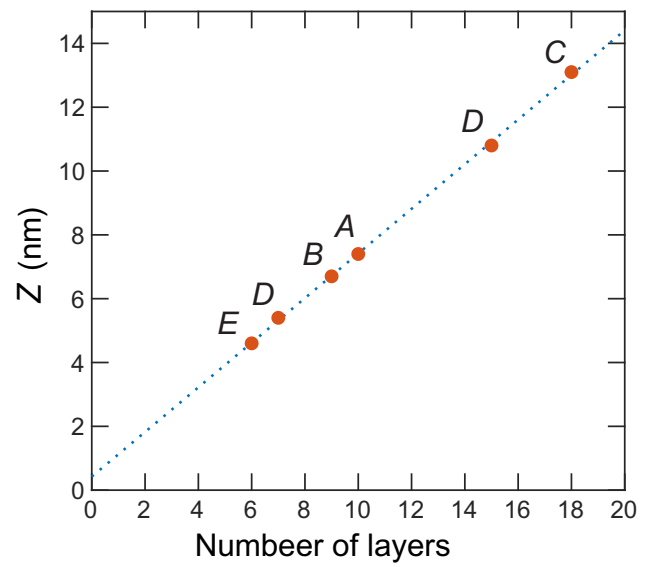

FIG. 7. AFM-determined thickness versus layer number. Each data point is labeled by its flake name (e.g., Flake A, Flake B, Flake C, ... ). Flake D contains two well-defined step heights.

Flake D displays similar trends as Flake C (18 layers), presented in the main text, such as vanishing dc magnetization (dense domains) and a Hopkinson peak in $\chi_{\text {ac }}$ below $T_{c}$ during FC, which should be generic to bulklike $\mathrm{CrBr}_{3}$ flakes [7].

For Flake E (six layers), the dc hysteresis measurement is performed at two separate locations along the edge to gauge the degree of local variation [Figs. 9(d) and $9(\mathrm{e})$ ]. The saturation magnetization is quantitatively similar between the two locations, but the exact switching fields and Barkausen jumps differ slightly. We also present an extended linecut into the interior of Flake $\mathrm{E}$ at $B_{\mathrm{dc}}^{E}=$ $76 \mathrm{mT}$ that directly locates domain walls [Fig. 9(f)]. Three domain walls are observed at locations where the stray
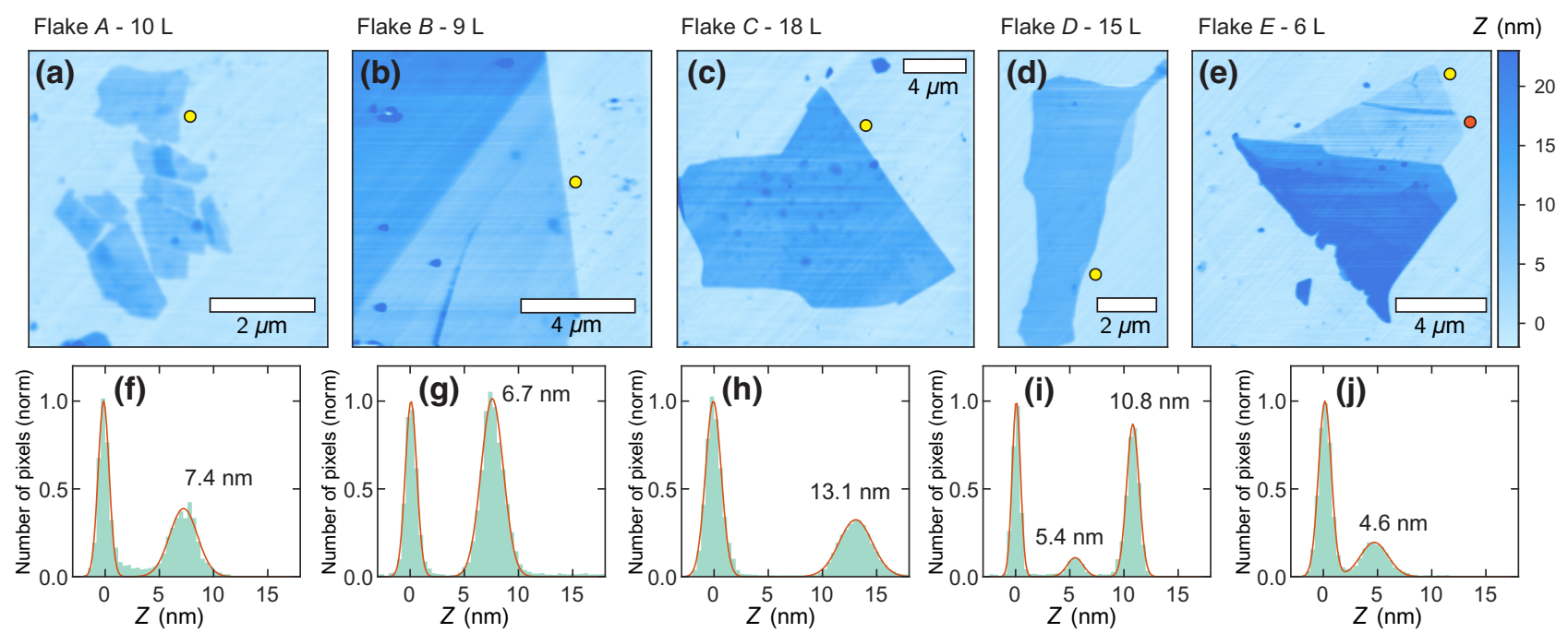

FIG. 8. (a)-(e) AFM topographies of $\mathrm{CrBr}_{3}$ flakes investigated in this work. (f)-(j) The step height histogram with Gaussian peak fitting is shown below the corresponding AFM topography and is used to determine the flake thickness. NV center magnetometry is predominantly performed at the location indicated by the yellow or red dot plotted in the AFM topography. For Flake E (six layers), the dc hysteresis is measured at two different locations along the edge of the flake. 
(a) Flake $C-18 \mathrm{~L}$

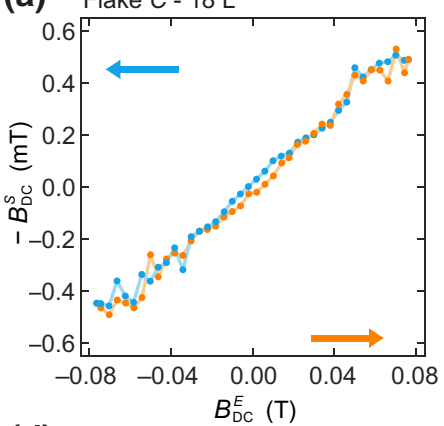

(d) Flake $E-6 \mathrm{~L}$

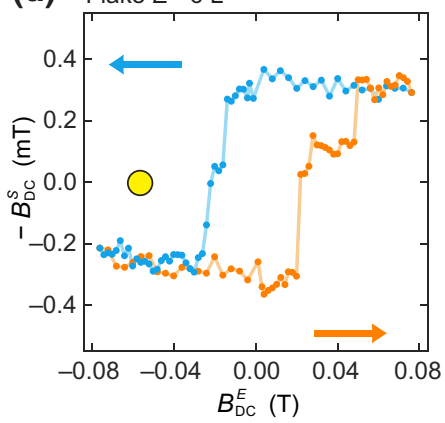

(b) Flake $D-15 \mathrm{~L}$

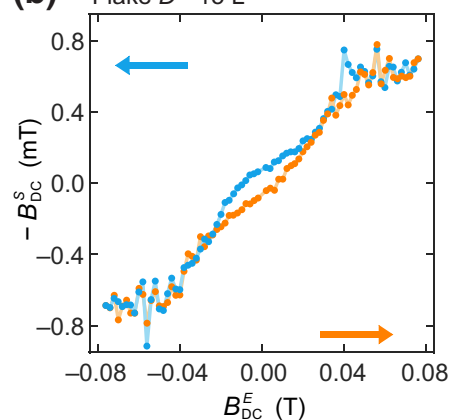

(e) Flake $E-6 L$

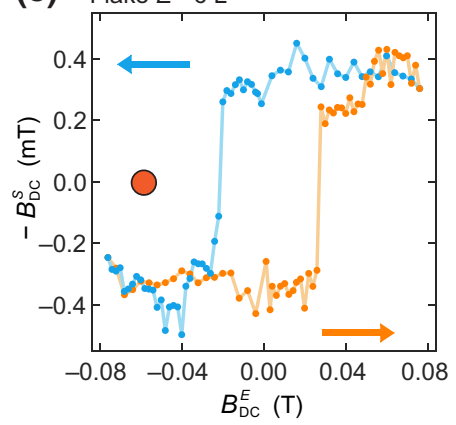

(c) Flake $D-15 \mathrm{~L}$

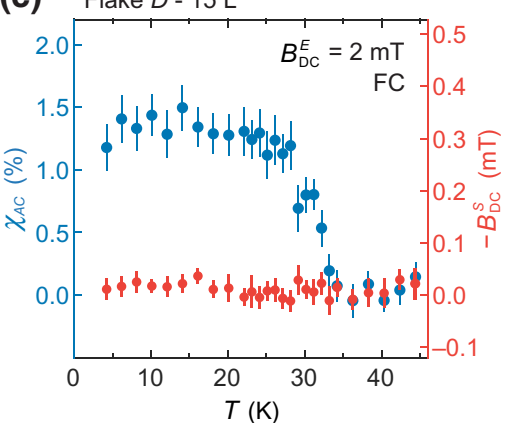

(f) Flake $E-6 \mathrm{~L}$

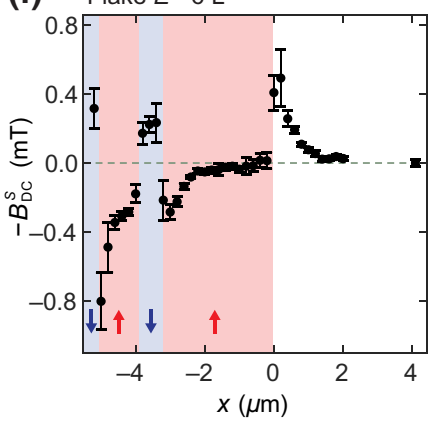

FIG. 9. Additional dc and ac measurements of $\mathrm{CrBr}_{3}$ flakes. (a),(b) Hysteresis curves for Flake C (a) and Flake D (b) measured at 4 K. (c) Field-cooling ac susceptibility $\chi_{\mathrm{ac}}$ (blue) for Flake D at $B_{\mathrm{dc}}^{E}=2 \mathrm{mT}$. The simultaneously measured dc magnetization $\left(\propto-B_{\mathrm{dc}}^{S}\right)$ is shown in red on the same plot. (d),(e) Hysteresis curves for Flake E measured at two different locations along the edge, as indicated in Fig. 8(e). (f) Extended linecut of the stray field across the right edge $(x=0)$ of Flake $\mathrm{E}$ that directly locates domain walls. Domains aligned (antialigned) with the bias field $B_{\mathrm{dc}}^{E}=76 \mathrm{mT}$ are shaded in red (blue).

field rapidly switches sign. While full 2D spatial imaging at optical spatial resolution is possible with our experimental setup [63], we refrain from extended measurements in the interior of the flakes to avoid light-induced oxidation of few-layer $\mathrm{CrBr}_{3}$.

\section{APPENDIX C: FIELD-GRADIENT BROADENING OF THE ENSEMBLE ODMR LINESHAPE}

Under the approximation of a semi-infinite, zero thickness dipole sheet, the vector stray field $\vec{B}^{S}=\left(B_{x}^{S}, B_{y}^{S}, B_{z}^{S}\right)$ due to a magnetic thin flake with out-of-plane polarization $(+z)$ is $[35,64]$

$$
\begin{aligned}
& B_{x}^{S}(\vec{r})=\frac{\mu_{0} \sigma_{z}}{2 \pi} \times \frac{z}{x^{2}+z^{2}}, \\
& B_{y}^{S}(\vec{r})=0, \\
& B_{z}^{S}(\vec{r})=-\frac{\mu_{0} \sigma_{z}}{2 \pi} \times \frac{x}{x^{2}+z^{2}},
\end{aligned}
$$

where $\vec{r}=(x, y, z)$, the semi-infinite dipole sheet resides in the $z=0$ plane for $x<0$ with the $y$ axis being its edge, $\mu_{0}$ is the vacuum magnetic permeability, and $\sigma_{z}$ is the areal magnetization density. With $B_{y}^{S}=0$ and a NV center axis in the $x-z$ plane, we can decompose the stray field in the coordinate system parallel and perpendicular to the NV axis, i.e., $\vec{B}^{S}=\left(B_{x}^{S}, B_{z}^{S}\right) \rightarrow\left(B_{\mathrm{NV} \|}^{S}, B_{\mathrm{NV} \perp}^{S}\right)$, where

$$
B_{\mathrm{NV} \|}^{S}(\vec{r})=\frac{\mu_{0} \sigma_{z}}{2 \pi} \cdot\left(\frac{z}{x^{2}+z^{2}} \sin (\theta)-\frac{x}{x^{2}+z^{2}} \cos (\theta)\right),
$$

$$
B_{\mathrm{NV} \perp}^{S}(\vec{r})=\frac{\mu_{0} \sigma_{z}}{2 \pi} \cdot\left(\frac{z}{x^{2}+z^{2}} \cos (\theta)+\frac{x}{x^{2}+z^{2}} \sin (\theta)\right) .
$$

Here, $\theta=54.74^{\circ}$ is the $\mathrm{NV}$ center polar angle \{i.e., NV axis is parallel to $[\sin (\theta), 0, \cos (\theta)]$ in $x y z$ coordinates\}, and in this particular sample $z=-60 \mathrm{~nm}$, as described in Appendix A. As explained in the main text, we fit the $4 \mathrm{~K}$ data in Fig. 3(e) (artifact ac signal induced by the flake's dc magnetic field perpendicular to the NV axis) to Eq. (C3) and fit the $26 \mathrm{~K}$ data (real ac magnetization signal of the sample) to Eq. (C2).

When the external dc field is perfectly aligned with the $\mathrm{NV}$ center axis, the ground-state spin $|0\rangle \rightarrow| \pm 1\rangle$ transition frequency is determined by a linear Zeeman splitting, i.e., $f_{ \pm}=D_{0} \pm \gamma_{e} \times B_{\mathrm{NV} \|}$, where $D_{0}=2.87 \mathrm{GHz}$ is the zero-field splitting, $\gamma_{e}=28 \mathrm{MHz} / \mathrm{mT}$ is the gyromagnetic ratio, and $B_{\mathrm{NV} \|}$ is the external field along the NV axis. With a magnetic flake present, the sample stray field $\vec{B}^{S}$ induces an additional frequency shift $\Delta f(\vec{r})$. In the ODMR 
spectrum, we consider the first-order approximation

$$
\Delta f(\vec{r})=-\gamma_{e} \times B_{\mathrm{NV} \|}^{S}(\vec{r})
$$

for the $|0\rangle \rightarrow|-1\rangle$ transition, where $\Delta f$ is linearly dependent on the parallel component of the local sample stray field. The field $B_{\mathrm{NV} \perp}^{S}$ does not affect the ODMR measurements within our experimental precision; however, we shall see that it induces a resolvable background signal in our ac sensing measurements (see Appendix E for details).

For ensemble NV samples, the green light reads out a collective signal of NV centers within the beam size. The ODMR lineshape maintains a narrow shape when the NV centers experience nearly uniform magnetic field (far from the flake), but near the edge of the flake where the magnetic field is rapidly changing spatially, the ODMR signal becomes broadened. For fixed $z$ depth, the shape of the ODMR dip measured at a location $\vec{r}=(x, y)$ relative the flake's edge at $x=0$ can be described by an integral of Lorentzian functions with varying center frequencies $f_{0}$ due to the local magnetic field, modulated by a Gaussian envelope that represents the density of NV centers probed over the beam size:

$$
I(f, \vec{r})=A \int d^{2} \overrightarrow{r^{\prime}} \frac{e^{-\left[\left(\overrightarrow{r^{\prime}}-\vec{r}\right) / \sqrt{2} \sigma\right]^{2}}}{2 \pi \sigma^{2}} \times \frac{(w / 2)^{2}}{\left[f-f_{0}\left(\overrightarrow{r^{\prime}}\right)\right]^{2}+(w / 2)^{2}} .
$$

Here the integral is over a circularly symmetric beam spot centered about $\vec{r}$. The integral $I(f, \vec{r})$ models the experimentally measured PL intensity with beam center at $\vec{r}$ as a function of the microwave frequency $f$. The beam width $\sigma$ is determined from single NV PL scans to be $0.17 \mu \mathrm{m}$. The zero-gradient linewidth $w$ for the Lorentzian lineshape is estimated to be $8 \mathrm{MHz}$ by measuring $\mathrm{NV}$ centers away from any flakes using the same microwave power settings. The center frequency $f_{0}\left(\overrightarrow{r^{\prime}}\right)$ as a function of position is determined by the stray field of the flake, using Eqs. (C2) and (C4). Since $f\left(\overrightarrow{r^{\prime}}\right)$ depends only on $x^{\prime}$, the coordinate perpendicular to the flake's edge, Eq. (C5) can be simplified to a $1 \mathrm{D}$ integral by integrating out the $y^{\prime}$ coordinate:

$$
I(f, x)=A \int d x^{\prime} \frac{e^{-\left[\left(x^{\prime}-x\right) / \sqrt{2} \sigma\right]^{2}}}{\sqrt{2 \pi} \sigma} \times \frac{(w / 2)^{2}}{\left[f-f_{0}\left(x^{\prime}\right)\right]^{2}+(w / 2)^{2}} .
$$

We fit the experimental ODMR traces as a function of the distance $x$ simultaneously to Eq. (C6). Explicitly, $z$, $\theta, \sigma$, and $w$ are fixed parameters determined from other measurements, $A$ is a free parameter that varies for each data set, and $\sigma_{z}$ is a common free parameter used for all data sets. An example of this fitting procedure is shown in Fig. 2(a) of the main text, where we determine $\sigma_{z}=$ $86 \pm 5 \mu_{b} / \mathrm{nm}^{2}$ for a nine-layer $\mathrm{CrBr}_{3}$ flake (Flake B).

\section{APPENDIX D: DETERMINATION OF THE AMPLITUDE OF THE ac FIELD $B_{\mathrm{ac}}^{T}$}

Our ac susceptibility technique is based on controlling the NV spin precession with an XY8- $N$ sequence while exciting the material with a synchronized ac magnetic field, similar in concept to the detection of pulsed photocurrents in monolayer $\mathrm{MoS}_{2}$ [28]. As shown in Fig. 3(a) in the main text, the sequence contains $8 N+2$ pulses on the NV center spin:

$$
\left(\frac{\pi}{2}\right)_{x}-\left[\pi_{x}-\pi_{y}-\pi_{x}-\pi_{y}-\pi_{y}-\pi_{x}-\pi_{y}-\pi_{x}\right]^{N}-\left(\frac{\pi}{2}\right)_{\text {proj }} .
$$

The $\pi$ pulses are separated by a free evolution time $\tau$, while the ac excitation field is resonant with this sequence: at zero lag, the ac field switches sign for every $\pi$ pulse [i.e., $\left.f_{\mathrm{ac}}=1 /(2 \tau)\right]$. Generally, the relative lag between the $\mathrm{XY} 8-N$ sequence and the ac excitation field is denoted as $t_{0}=\delta / \pi \times \tau$, where $\delta$ is the phase delay in radians. Each $\pi_{j}$ pulse flips the NV superposition state around the $j$ axis on the Bloch sphere by a half-circle [e.g., $(1 / \sqrt{2})(|0\rangle+$ $\left.e^{-i \varphi}|-1\rangle\right)$ is turned into $(1 / \sqrt{2})\left(|0\rangle+e^{i \varphi}|-1\rangle\right)$ by a $\pi_{x}$ pulse, negating the phase accumulated prior to the pulse]. Thus, the net action of the $\pi$ pulses is to rectify the phase accumulation due to the ac field, which would otherwise vanish by time integration. The rotation around orthogonal axes in succession decouples pulse errors.

The total accumulated phase $\varphi_{\text {end }}$ at the end of the evolution is optically readout after a $(\pi / 2)_{x}$ projection pulse. The measured NV center PL is proportional to $X_{P}(\delta)=$ $A \cos \left[\varphi_{\text {end }}(\delta)\right]$, where $X_{P}$ is the $x$ projection of the final superposition state on the Bloch sphere and $A$ is the measurement contrast. We first consider that the external dc field is perfectly aligned along the NV center axis such that the frequency shift of the NV spin state is due to only the component of the ac field parallel to the NV axis:

$$
\varphi_{\mathrm{end}}(\delta)=\int_{t_{0}}^{t_{0}+8 N \tau} 2 \pi \gamma_{e} \times \widetilde{B}_{\mathrm{NV} \|}^{T}(t) \times M\left(t-t_{0}\right) d t
$$

Here $t_{0}=\delta \times \tau / \pi$ and the modulation function representing the effect of the $\pi$ pulses is

$$
M(t)= \begin{cases}-1, & 0 \leqslant \bmod (t / \tau-0.5,2)<1 \\ +1, & 1 \leqslant \bmod (t / \tau-0.5,2)<2\end{cases}
$$

Note that in the appendices any tilded symbol refers to the amplitude of an ac field. Here $\widetilde{B}_{\mathrm{NV} \|}^{T}(t)$ is the total ac field parallel to the NV center axis, consisting of the excitation 
coil field $\widetilde{B}_{\mathrm{NV} \|}^{C}$ and the sample ac response $\widetilde{B}_{\mathrm{NV} \|}^{S}$,

$$
\begin{aligned}
\widetilde{B}_{\mathrm{NV} \|}^{T}(t)= & \widetilde{B}_{\mathrm{NV} \|}^{C}(t)+\widetilde{B}_{\mathrm{NV} \|}^{S}(t) \\
= & \widetilde{B}_{\mathrm{NV} \|}^{C} \cdot\left[(1+\kappa) \cdot \cos \left(\frac{\pi}{\tau} \times t\right)\right. \\
& \left.+\kappa^{\prime} \sin \left(\frac{\pi}{\tau} \times t\right)\right],
\end{aligned}
$$

where $\kappa$ and $\kappa^{\prime}$ are proportional to real and imaginary parts of the complex ac susceptibility of the sample. By integrating Eq. (D1) with Eqs. (D2) and (D3) substituted in, the normalized $x$ projection of the final state $X_{P}=\cos \left[\varphi_{\text {end }}(\delta)\right]$ as a function of the phase delay $\delta$ is

$$
X_{P}(\delta)=\cos \left[\Phi_{\mathrm{NV}} \times \cos \left(\delta-\delta_{0}\right)\right]
$$

where

$$
\begin{aligned}
\Phi_{\mathrm{NV}} & =2 \pi \gamma_{e} \times 2 / \pi \times 8 N \tau \times\left|\widetilde{B}_{\mathrm{NV} \|}^{C}\right| \sqrt{(1+\kappa)^{2}+\kappa^{\prime 2}} \\
& =32 \gamma_{e} \times N \tau \times\left|\widetilde{B}_{\mathrm{NV} \|}^{T}\right|, \\
\delta_{0} & =\arctan \left(\frac{\kappa^{\prime}}{1+\kappa}\right) .
\end{aligned}
$$

We fit our experimental data to Eq. (D4) to determine $\Phi_{\mathrm{NV}}$, referred to as the maximum precession angle, and use Eq. (D5) to convert $\Phi_{\mathrm{NV}}$ to $\left|\widetilde{B}_{\mathrm{NV} \|}^{T}\right|$. If the imaginary component of the ac susceptibility is negligible, as in our case, then $\delta_{0} \rightarrow 0$ and $\widetilde{B}_{\mathrm{NV} \|}^{T} \rightarrow \widetilde{B}_{\mathrm{NV} \|}^{C} \cdot(1+\kappa)$. For brevity of notation, $\widetilde{B}_{\mathrm{NV} \|}^{T}$ is denoted as $B_{\mathrm{ac}}^{T}$ in the main text, $\widetilde{B}_{\mathrm{NV} \|}^{C}$ as $B_{\mathrm{ac}}^{C}$, etc.

Equation (D4) oscillates rapidly in $\delta$ when $\Phi_{\mathrm{NV}}$ is large. It is time consuming to map the whole fringe pattern, so $\delta$ is only swept around a small range around $\delta=0$. However, when $\Phi_{\mathrm{NV}}$ is large, the $X_{P}(\delta)$ fringes are almost invariant near $\delta=0$ when $\Phi_{\mathrm{NV}}$ differs by $\pm 2 n \pi$, where $n$ is a small integer. This suggests that the restricted $\delta$ sweep can only determine $\Phi_{\mathrm{NV}}$ modulo $2 \pi$. We illustrate
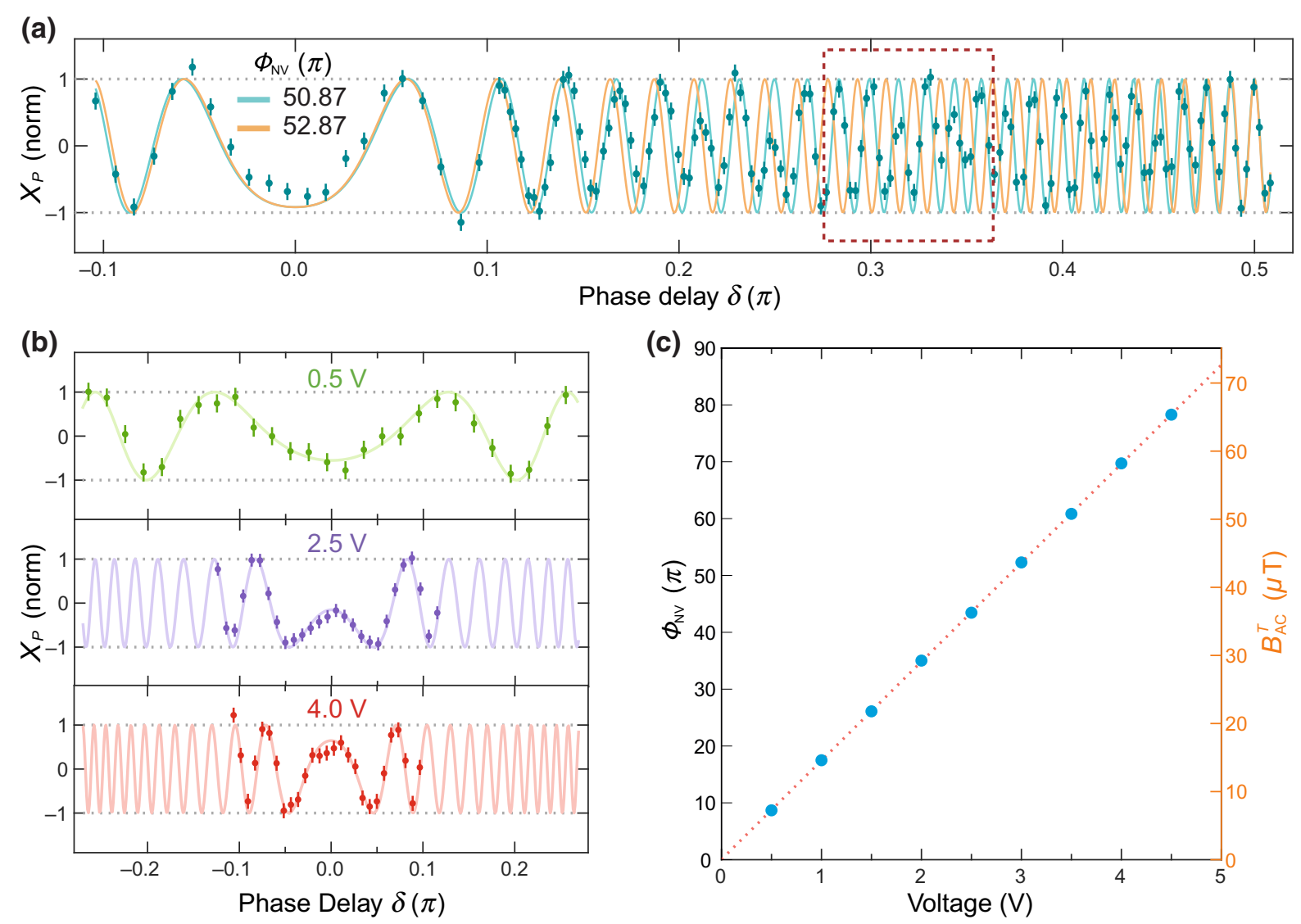

FIG. 10. Determining the absolute magnitude of $\Phi_{\mathrm{NV}}$. (a) Full fringe pattern of $X_{P}(\delta)$ (teal markers) and its best fit curve (teal line). The expected behavior of $X_{P}(\delta)$ for $\Phi_{\mathrm{NV}}$ offset by $2 \pi$ is also plotted (orange line), showing disagreement with the data in the boxed region. (b) The $X_{P}(\delta)$ curves for increasing amplitudes of the coil field (controlled by the voltage applied to the coil). (c) Fitted $\Phi_{\mathrm{NV}}$ versus coil voltage shows a precise linear dependence and confirms the correct assignment of the $2 \pi$ branch for each voltage. 
this in Fig. 10(a) for a simulation of $\Phi_{\mathrm{NV}}=50.87 \pi$ and $\Phi_{\mathrm{NV}}=52.87 \pi$. However, the change in $\Phi_{\mathrm{NV}}$ due to the sample response per temperature or magnetic field increment is much smaller than $2 \pi$. If we first determine which $2 \pi$ branch of $\Phi_{\mathrm{NV}}$ we start in, then we can track these incremental changes continuously by restricting the fit to the appropriate $2 \pi$ branch.

There are two ways to initially determine the absolute magnitude of $\Phi_{\mathrm{NV}}$. The first is to simply measure $X_{P}(\delta)$ for $\delta$ spanning more than $\pi / 2$ once prior to a temperature sweep. Figure 10(a) shows an exemplary full $\delta$ sweep. On this full range, the teal experimental data clearly fit to $\Phi_{\mathrm{NV}}=50.87 \pi$ and not to $\Phi_{\mathrm{NV}}=52.87 \pi$, as is evident in the fast oscillating region highlighted by the dashed rectangle.

The second method is to measure $X_{P}(\delta)$ at different amplitudes of the coil field, controlled by the voltage output of the ac generator. Far from any flakes, $\Phi_{\mathrm{NV}}$ should be proportional to the current (voltage) in the coil. As shown in Figs. 10(b) and 10(c), the fringe pattern is sampled for increasing voltages, where, for the lowest voltage, there is no $2 \pi$ ambiguity in determining $\Phi_{\mathrm{NV}}$. The linear scaling of $\Phi_{\mathrm{NV}}$ versus voltage shown in Fig. 10(c) confirms the correct $2 \pi$ assignments. Hence, by using these methods to determine the initial $2 \pi$ branch for $\Phi_{\mathrm{NV}}$, we can take a restricted set of data around $\delta=0$ during a sweep, but still track the absolute magnitude of $\widetilde{B}_{\mathrm{NV} \|}^{T}$ without ambiguity.

\section{APPENDIX E: ac BACKGROUND INDUCED BY dc MAGNETIZATION OF THE FLAKE}

In Fig. 11, we present the nonbackground corrected data for $\chi_{\mathrm{ac}} \equiv-\widetilde{B}_{\mathrm{NV} \|}^{S} / \widetilde{B}_{\mathrm{NV} \|}^{C}$ (blue) alongside the simultaneously acquired dc sample stray field $B_{\mathrm{NV} \|}^{S}$ (orange) for all temperature sweeps on Flake A [Figs. 3(f) and 4(b)-4(d) of the main text]. The similarity in the shapes of the ac

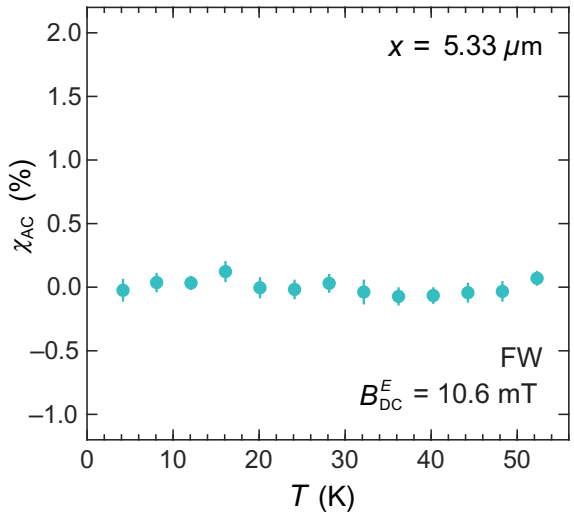

FIG. 12. Coil field versus temperature. The susceptibility $\chi_{\mathrm{ac}}$ up to $52 \mathrm{~K}$ measured for NV centers far from Flake A, representing the bare coil field. It shows no systematic change due to the drift of the coil relative to the sample or the change in the current in the coil in this temperature range.

and dc data for the FW sweep from the saturated state [Fig. 11(a)] suggests that somehow the dc field of the magnetic flake influences the background subtraction of the coil field, since $\chi_{\mathrm{ac}}$ should vanish for the saturated state at low temperature. By measuring $\mathrm{NV}$ centers in isolation from the flake, we verify in Fig. 12 that the coil field itself (reflected by its component parallel to the NV axis, $\widetilde{B}_{\mathrm{NV} \|}^{C}$ ) does not actually change with temperature due to drift or resistance change of the coil. In this section, we explain theoretically that the additional background contribution to the ac signal stems from the changing alignment between the total dc magnetic field and the NV center axis, which couples the perpendicular component of the coil field $\widetilde{B}_{\mathrm{NV} \perp}^{C}$ into the ac measurement.

In a rotating-frame precession (Ramsey or XY8- $N$ ) measurement, the phase accumulation of the NV
Flake $A-10 \mathrm{~L}$

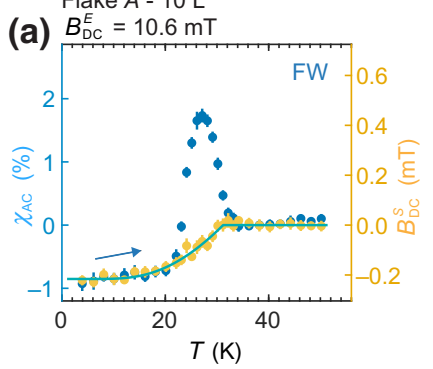

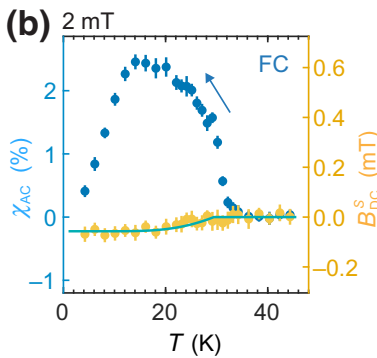

(c)

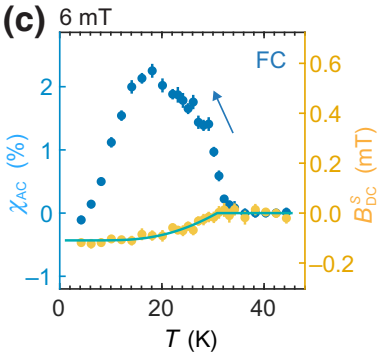

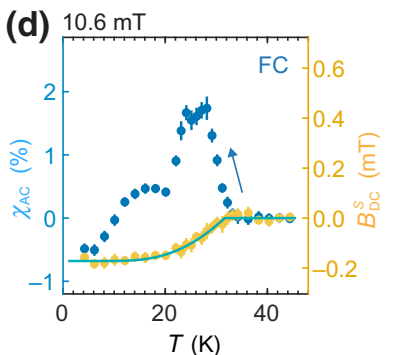

FIG. 11. Uncorrected $\chi_{\mathrm{ac}}$ data for Flake A. (a) FW sweep at $B_{\mathrm{dc}}^{E}=10.6 \mathrm{mT}$. The shape of negative (artifact) $\chi_{\mathrm{ac}}$ background at low temperature (blue) tracks the simultaneously measured dc stray field of the flake, $B_{\mathrm{dc}}^{S}$ (orange). The signal due to the physical ac magnetization response in the flake is therefore the deviation from a temperature-dependent baseline proportional to the flake's dc field (orange). (b)-(d) FC sweeps at $B_{\mathrm{dc}}^{E}=2 \mathrm{mT}$ (b), $6 \mathrm{mT}$ (c), and $10.6 \mathrm{mT}$ (d). To determine the baseline corrected data for Flake A presented in the main text [Fig. 3(f) inset and Figs. 4(b)-4(d)], $B_{\mathrm{dc}}^{S}$ below $T_{c}$ is first fit to a spin wave model $B_{\mathrm{dc}}^{S} \propto S-\left(k_{B} T^{\beta} / 2 \pi J S\right) e^{-\Delta_{0} / k_{B} T}$ to smooth noise [10]. The fitted curves (shown here in green) are then scaled by the same constant of proportionality, determined by setting the $\chi_{\mathrm{ac}}$ data in (a) to zero at low temperature, and subtracted from their respective uncorrected $\chi_{\mathrm{ac}}$. No background correction for $\chi_{\mathrm{ac}}$ is performed for Flake C [Fig. 4(a)] or D [Fig. 9(c)] since the dc field from the flake is vanishing. 

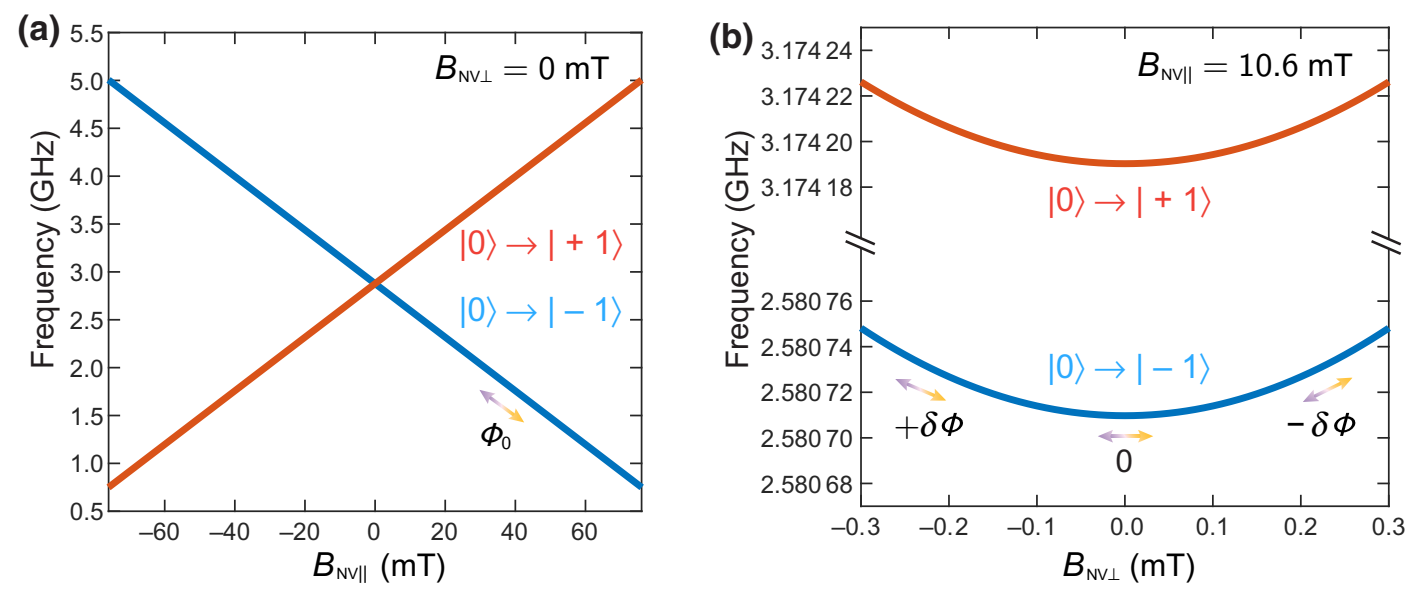

FIG. 13. (a) NV center spin energies as a function of $B_{\mathrm{NV} \|}$, parallel to the NV axis, with zero off-axis field $B_{\mathrm{NV} \perp}$. The slope of the energy versus field is not field dependent. (b) NV center spin energies as a function of $B_{\mathrm{NV} \perp}$, perpendicular to the NV axis, with fixed $B_{\mathrm{NV} \|}$. The slope is proportional to $B_{\mathrm{NV} \perp}$, although it is much smaller in absolute magnitude than (a). The maximum precession angle $\Phi_{\mathrm{NV}}$ in an XY8-N sequence is proportional to the sum of the ac field amplitude in each direction multiplied by its respective slope.

superposition state $(1 / \sqrt{2})\left(|0\rangle+e^{i \varphi}| \pm 1\rangle\right)$ comes from the energy detuning of $| \pm 1\rangle$ relative to the frequency of the microwave pulses. For an ac field, the NV center experiences a periodic energy detuning, which is rectified by the $\mathrm{XY} 8-N$ sequence. The net precession angle is thus proportional to the shift in energy of the $| \pm 1\rangle$ states due to the additional ac magnetic field. The transition frequency of $|0\rangle \rightarrow| \pm 1\rangle$ in an arbitrary static field $\vec{B}$ can be explicitly calculated [65] as

$$
\begin{aligned}
f_{ \pm}= & D_{0}+\frac{3\left(\gamma_{e} B\right)^{2}}{2 D_{0}} \sin ^{2} \theta_{B} \\
& \pm \gamma_{e} B \cos \theta_{B} \sqrt{1+\left(\frac{\gamma_{e} B}{2 D_{0}}\right)^{2} \tan ^{2} \theta_{B} \sin ^{2} \theta_{B}} \\
\approx & D_{0}+\frac{3 \gamma_{e}^{2}}{2 D_{0}}\left(B_{\mathrm{NV} \perp}\right)^{2} \pm \gamma_{e} B_{\mathrm{NV} \|},
\end{aligned}
$$

where $B=\sqrt{B_{\mathrm{NV} \perp}^{2}+B_{\mathrm{NV} \|}^{2}}$ and $\tan \theta_{B}=B_{\mathrm{NV} \perp} / B_{\mathrm{NV} \|}$. In the second line, we have kept terms of order $\theta_{B}^{2}$ and omitted terms of order $\theta_{B}^{4}$, since the misalignment introduced by the flake is expected to be small. The shape of these energy dispersions is plotted in Fig. 13.

When a small ac field is now superposed on top of the static field $\vec{B}$ (i.e., $B_{\mathrm{NV} \|} \rightarrow B_{\mathrm{NV} \|}+\widetilde{B}_{\mathrm{NV} \|}^{T}$ and $B_{\mathrm{NV} \perp} \rightarrow$ $B_{\mathrm{NV} \perp}+\widetilde{B}_{\mathrm{NV} \perp}^{T}$ ), the change in the transition frequency becomes

$$
\begin{aligned}
\Delta f_{ \pm} & =\frac{\partial f_{ \pm}}{\partial B_{\mathrm{NV} \perp}} \times \widetilde{B}_{\mathrm{NV} \perp}^{T}+\frac{\partial f_{ \pm}}{\partial B_{\mathrm{NV} \|}} \times \widetilde{B}_{\mathrm{NV} \|}^{T} \\
& =\frac{3 \gamma_{e}^{2}}{D_{0}}\left(B_{\mathrm{NV} \perp} \times \widetilde{B}_{\mathrm{NV} \perp}^{T}\right) \pm \gamma_{e} \widetilde{B}_{\mathrm{NV} \|}^{T}
\end{aligned}
$$

The precession angle $\Phi_{\mathrm{NV}}$ is proportional to the total energy shift $\Delta f_{ \pm}$induced by the ac field; hence, it is contributed by the sum of two parts: $\Phi_{\mathrm{NV} \|} \propto \widetilde{B}_{\mathrm{NV} \|}^{T}$ and $\Phi_{\mathrm{NV} \perp} \propto B_{\mathrm{NV} \perp} \times \widetilde{B}_{\mathrm{NV} \perp}^{T}$. The contribution $\Phi_{\mathrm{NV} \|}$ is independent of the value of the dc field, but the contribution $\Phi_{\mathrm{NV} \perp}$ is directly proportional to the transverse dc field $B_{\mathrm{NV} \perp}$. Near the edge of the flake where our measurements are performed, $B_{\mathrm{NV} \perp}$ changes as the flake's magnetization changes with temperature. We carefully align our magnet so that the transverse dc field $B_{\mathrm{NV} \perp}$ is dominated by the contribution of the flake, but our ac coil field cannot be aligned along the $\mathrm{NV}$ axis $\left(\widetilde{B}_{\mathrm{NV} \perp}^{T}\right.$ is significant). Hence, Eq. (E2) directly explains why an additional contribution proportional to $B_{\mathrm{NV} \perp} \times \widetilde{B}_{\mathrm{NV} \perp}^{T} \approx B_{\mathrm{NV} \perp} \times \widetilde{B}_{\mathrm{NV} \perp}^{C} \propto M(T) \cdot \widetilde{B}_{\mathrm{NV} \perp}^{C}$

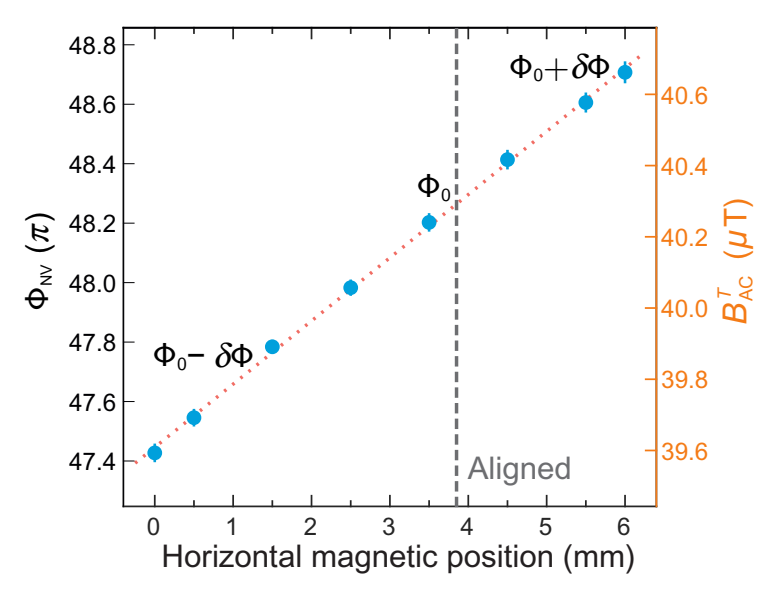

FIG. 14. Linear dependence of the measured maximum precession angle $\Phi_{\mathrm{NV}}$ on $B_{\mathrm{NV} \perp}$, introduced by deliberately translating our magnet in the horizontal direction about the optimal alignment at approximately $3.8 \mathrm{~mm}$. 
is superimposed onto the temperature-dependent ac susceptibility data.

As a final check, we confirm this effect for NV centers isolated from any flakes by purposely changing $B_{\mathrm{NV} \perp}$ through translating our permanent magnet, simulating the effect of the flake. As shown in Fig. 14, the maximum precession angle $\Phi_{\mathrm{NV}}$ indeed changes linearly versus small horizontal displacements of the permanent magnet, which is approximately linearly proportional to $B_{\mathrm{NV} \perp}$. This measurement also verifies that the signs of the change in $\Phi_{\mathrm{NV}}$ for the dc field produced by the magnetized flake and by the magnet displacement are consistent with each other.

\section{APPENDIX F: DETERMINATION OF THE QUANTITATIVE ac SUSCEPTIBILITY $\chi_{\mathrm{ac}}^{\|}$}

Because of strong out-of-plane magnetic anisotropy, $\mathrm{CrBr}_{3}$ forms $180^{\circ}$ domain walls below $T_{c}$ [20]. In this case, the ac susceptibility should be dominated by DWDs, which are only sensitive to the component of the coil field parallel to the easy axis ( $c$ axis of $\mathrm{CrBr}_{3}$ or the $z$ direction in the lab frame) [66]. The coil field perpendicular to the $c$ axis can drive magnetization rotation away from the easy axis, but this susceptibility is strongly suppressed by the magnetic anisotropy [50] and by the small amplitude of the coil field in this direction in our experimental geometry. Hence, our measurements predominantly detect the parallel ac susceptibility $\chi_{\text {ac }}^{\|}$, defined as the magnetic moment change per unit coil field $\widetilde{B}_{z}^{C}$ along the $z$ direction, per mole of $\mathrm{Cr}$ ions. We estimate the excitation amplitude $\widetilde{B}_{z}^{C}$ from the measured $\widetilde{B}_{N V \|}^{C}$. Figure 15 diagrams the orientation of the coil field in the $x-z$ plane, containing the out-of-plane $z$ axis and the NV center axis. The angle $\alpha$ between the $z$ axis and the component of the coil field $\widetilde{B}_{x z}^{C}$ in the $x-z$ plane is estimated from the location of the particular flake within the coil, by taking reasonable values for

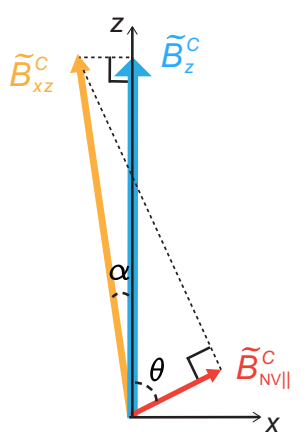

FIG. 15. Illustration of external ac field geometry. Here $\widetilde{B}_{x y}^{C}$ (orange) is the direction of the coil's ac field in the $x-z$ plane at the location of the sample. Its projection on the NV center axis $\widetilde{B}_{\mathrm{NV} \|}^{C}$ (red) is directly measured. We compute the out-of-plane projection of the coil field $\widetilde{B}_{z}^{C}$ (blue) and use it as the effective driving field amplitude to calculate the ac susceptibility $\chi_{\mathrm{ac}}^{\|}$parallel to the $c$ axis. the coil radius $(0.5 \mathrm{~mm})$ and the height of the coil above the diamond $(0.2 \mathrm{~mm})$. From the geometry shown, we deduce that $\widetilde{B}_{z}^{C}=\widetilde{B}_{\mathrm{NV} \|}^{C} \cdot[\cos \alpha / \cos (\theta+\alpha)]$, where $\widetilde{B}_{\mathrm{NV} \|}^{C}$ is directly measured, $\theta=54.7^{\circ}$ is the angle of the NV axis, and $|\alpha|<10^{\circ}$ for all data presented.

The molar ac susceptibility $\chi_{\mathrm{ac}}^{\|}$can be expressed as

$$
\chi_{\mathrm{ac}}^{\|}=\Delta \sigma_{z} /\left(\rho \times t \times\left|\widetilde{B}_{z}^{C}\right|\right)
$$

where $\Delta \sigma_{z}$ is the amplitude of the ac magnetization change in emu/area (or, equivalently, $\mu_{B} /$ area), $\rho=$ $0.0146 \mathrm{~mol} \mathrm{~cm}^{-3}$ is the molar density for $\mathrm{CrBr}_{3}, t$ is the thickness of the flake, and $\left|\widetilde{B}_{z}^{C}\right|$ is the amplitude of the ac driving field. The notion of a single value for $\chi_{\mathrm{ac}}^{\|}$treats $\Delta \sigma_{z}$ as the spatially averaged magnetization change over the sample. The spatial details of the source of the susceptibility can in general influence the stray field $\widetilde{B}_{\mathrm{NV} \|}^{S}$ measured at a particular location by NV magnetometry. However, we find that the stray field outside the flake provides a convergent estimate for the spatially averaged magnetization change when the individual sources of susceptibility are relatively dense on the length scale of the distance between the NV center probe and the flake's edge.

To illustrate, we simulate in Fig. 16 the stray field for DWDs of stripe domains with $100 \mathrm{~nm}$ width, as consistent with electron microscopy imaging of bulk $\mathrm{CrBr}_{3}$ [44]. We assume that domains aligned with the excitation field expand by $10 \mathrm{~nm}$ at the expense of antialigned domains. The amplitude of the DWD $(10 \mathrm{~nm})$ is not meant to be realistic for our ac driving field amplitude, but to facilitate simulations over a $24 \mu \mathrm{m}$ by $24 \mu \mathrm{m}$ region using $5 \mathrm{~nm}$ spatial resolution. The spatial distribution for the magnetization change $\Delta \sigma_{\mathrm{DW}}$ is therefore $10 \mathrm{~nm}$ stripes of amplitude $2 \sigma_{S}$ at the locations of the domain walls, forming an assumed $10^{\circ}$ angle to the edge of the flake [Fig. 16(a)]. Here, we take $\sigma_{S}=14.8 \mu_{B} / \mathrm{nm}^{2}$ as the saturation magnetization of monolayer $\mathrm{CrBr}_{3}$. Alternatively, we can consider distributing the same total magnetization change due the DWDs homogeneously over the flake in Fig. 16(c). In this case, the flake's magnetization changes by $\Delta \sigma_{\text {hom }}=2.96 \mu_{B} / \mathrm{nm}^{2}$ everywhere.

Figure 16(b) presents the simulated stray field $\Delta B_{\mathrm{DW}}$ along the NV center axis at an NV depth of $z=-60$ $\mathrm{nm}$ for the distribution $\Delta \sigma_{\mathrm{DW}}$ using Fourier propagation [18]. Linecuts for the stray field across the edge of the flake are shown in Fig. 16(d) corresponding to $\Delta \sigma_{\mathrm{DW}}$ for domain walls at three different angles (solid lines) and for the homogeneously distributed magnetization change $\Delta \sigma_{\text {hom }}$ (dashed lines). Independent of the exact orientation of the domain walls, the ratio for the stray fields $\Delta B_{\mathrm{DW}} / \Delta B_{\text {hom }}$ converges to 1 as the distance $x$ to the edge of the flake increases, and is within $2 \%$ difference by $x=0.3 \mu \mathrm{m}$. Hence, these simulations indicate that the spatially averaged magnetization change can be estimated 

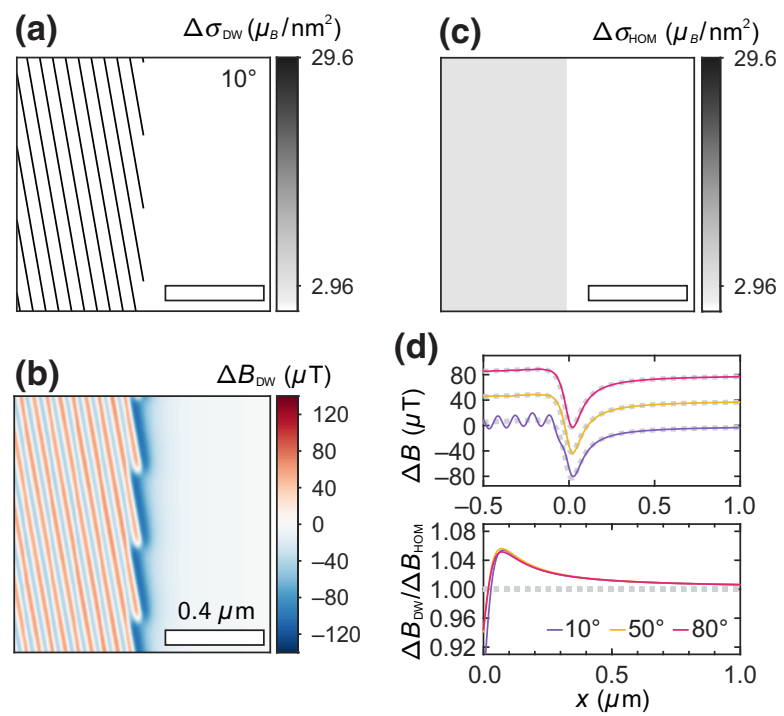

FIG. 16. (a) Spatial distribution of the magnetization change $\Delta \sigma_{\text {DW }}$ for DWDs of stripe domains. The domain walls form a $10^{\circ}$ angle to the edge of the flake with spacing of $100 \mathrm{~nm}$, and are assumed to move by $10 \mathrm{~nm}$ under an excitation field. (b) Simulated stray field $\Delta B_{\mathrm{DW}}$ along the NV center axis due to $\Delta \sigma_{\mathrm{DW}}$. (c) Spatial distribution of a homogeneous magnetization change $\Delta \sigma_{\text {hom }}$, equivalent to spatial average of $\Delta \sigma_{\mathrm{DW}}$. (d) Top: linecuts of the stray field $\Delta B$ versus the distance $x$ perpendicular to the flake's edge. The field produced by three different angles for $\Delta \sigma_{\mathrm{DW}}$ are shown as the solid lines and for $\Delta \sigma_{\text {hom }}$ as the dashed lines. The linecuts are offset for clarity and are Gaussian averaged in the $y$ direction, parallel to the flake, over our optical beam size. Bottom: ratio of the stray field due to the particular domain wall distribution ( $\left.\Delta B_{\mathrm{DW}}\right)$ to that produced by uniformly distributing the total magnetization change over area $\left(\Delta B_{\text {hom }}\right)$. The ratio approaches one as $x$ increases, independent of the exact spatial distribution.

by assuming that the stray field is produced by a uniform, semi-infinite magnetized sheet. The accuracy of this estimation improves when the source of the susceptibility is densely distributed compared to the distance between the NV center and the flake. Hence, the accuracy of our quantitative estimation is highest when the domains are small, which is experimentally signaled by a vanishing or small de stray field at the NV center location. The limit is approached by Flake $\mathrm{C}$ and Flake $\mathrm{A}$ under field cooling at the smallest dc field [Figs. 4(a) and 4(b) of the main text].

For a uniformly magnetized sheet, we can calculate $\Delta \sigma_{z}$ from the measured ac stray field $\widetilde{B}_{\mathrm{NV} \|}^{S}$ via

$$
\begin{aligned}
\widetilde{B}_{\mathrm{NV} \|}^{S}= & \frac{\mu_{0} \Delta \sigma_{z}}{2 \pi}\left(\frac{z}{x^{2}+z^{2}} \sin (\theta) \cos \left(\phi_{\text {edge }}\right)\right. \\
& \left.-\frac{x}{x^{2}+z^{2}} \cos (\theta)\right)
\end{aligned}
$$

where $z=-60 \mathrm{~nm}, x$ is the perpendicular distance of the NV center to the flake's edge (determined from a linecut (a)

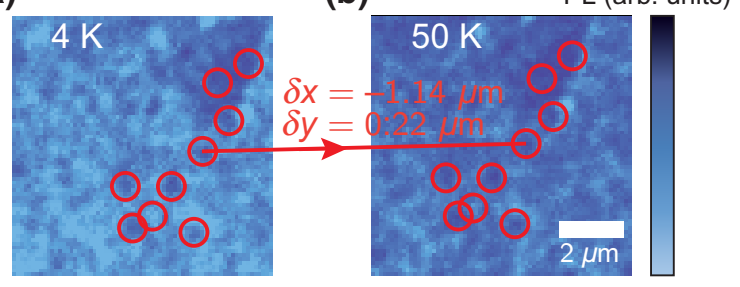

FIG. 17. Drift correction. (a) PL map at 4 K. (b) PL map at 50 K. Common features detected with the SIFT algorithm are encircled. The arrow linking one of the pairs is shown as a guide to the eye. We apply a drift correction corresponding to the average displacement of all pairs of common features.

of the flake's dc field), $\theta=54.7^{\circ}$ is the polar angle of the $\mathrm{NV}$ axis, and $\phi_{\text {edge }}$ is the azimuthal angle of the flake's edge relative to the NV center axis [see the AFM images in Fig. 8(e); the NV center axis projection is along the horizontal direction in these AFM scans]. For fixed NV center location during a sweep (see Appendix $\mathrm{G}$ about drift correction), $\widetilde{B}_{\mathrm{NV} \|}^{S}=K \times \Delta \sigma_{z}$, where all geometric factors are captured by $K$. Using the determined $\Delta \sigma_{z}$, we calculate $\chi_{\mathrm{ac}}^{\|}$, the susceptibility in the spatially averaged sense, via Eq. (F1).

\section{APPENDIX G: DRIFT CORRECTION}

Our measurements are sensitive to location of the probed NV centers relative to the flake's edge, as is evident from Figs. 2(b) and 3(e) of the main text for the dc and ac measurement modes, respectively. Hence, when changing the magnetic field or temperature, it is very important to compensate for drifts with well below $1 \mu \mathrm{m}$ accuracy. For all of the data we acquired, whenever the temperature or magnetic field is changed, a PL map of the NV ensemble is taken and then compared to a standard reference PL map of the same area. The comparison is realized with the scale-invariant feature transform (SIFT) function in the OpenCV PYTHON package to detect and match key features, as shown in Fig. 17. The drift is then determined by taking an average of the vector displacements between each pair of features. Through this method, the error in the drift correction is estimated to be approximately $50 \mathrm{~nm}$.

[1] K. S. Burch, D. Mandrus, and J. G. Park, Magnetism in two-dimensional van der Waals materials, Nature 563, 47 (2018).

[2] C. Gong and X. Zhang, Two-dimensional magnetic crystals and emergent heterostructure devices, Science $\mathbf{3 6 3}$, eaav4450 (2019).

[3] K. F. Mak, J. Shan, and D. C. Ralph, Probing and controlling magnetic states in 2D layered magnetic materials, Nat. Rev. Phys. 1, 646 (2019). 
[4] M. Buchner, K. Höfler, B. Henne, V. Ney, and A. Ney, Tutorial: Basic principles, limits of detection, and pitfalls of highly sensitive SQUID magnetometry for nanomagnetism and spintronics, J. Appl. Phys. 124, 161101 (2018).

[5] B. Huang, G. Clark, E. Navarro-Moratalla, D. R. Klein, R. Cheng, K. L. Seyler, D. Zhong, E. Schmidgall, M. A. McGuire, D. H. Cobden, W. Yao, D. Xiao, P. JarilloHerrero, and X. Xu, Layer-dependent ferromagnetism in a van der Waals crystal down to the monolayer limit, Nature 546, 270 (2017).

[6] C. Gong, L. Li, Z. Li, H. Ji, A. Stern, Y. Xia, T. Cao, W. Bao, C. Wang, Y. Wang, Z. Q. Qiu, R. J. Cava, S. G. Louie, J. Xia, and X. Zhang, Discovery of intrinsic ferromagnetism in two-dimensional van der Waals crystals, Nature 546, 265 (2017).

[7] C. Jin, Z. Tao, K. Kang, K. Watanabe, T. Taniguchi, K. F. Mak, and J. Shan, Imaging and control of critical fluctuations in two-dimensional magnets, Nat. Mater. 19, 1290 (2020).

[8] X. X. Zhang, L. Li, D. Weber, J. Goldberger, K. F. Mak, and J. Shan, Gate-tunable spin waves in antiferromagnetic atomic bilayers, Nat. Mater. 19, 838 (2020).

[9] K. L. Seyler, D. Zhong, D. R. Klein, S. Gao, X. Zhang, B. Huang, E. Navarro-Moratalla, L. Yang, D. H. Cobden, M. A. McGuire, W. Yao, D. Xiao, P. Jarillo-Herrero, and X. Xu, Ligand-field helical luminescence in a $2 \mathrm{D}$ ferromagnetic insulator, Nat. Phys. 14, 277 (2018).

[10] Z. Zhang, J. Shang, C. Jiang, A. Rasmita, W. Gao, and T. $\mathrm{Yu}$, Direct photoluminescence probing of ferromagnetism in monolayer two-dimensional $\mathrm{CrBr}_{3}$, Nano Lett. 19, 3138 (2019).

[11] D. Ghazaryan, M. T. Greenaway, Z. Wang, V. H. Guarochico-Moreira, I. J. Vera-Marun, J. Yin, Y. Liao, S. V. Morozov, O. Kristanovski, A. I. Lichtenstein, M. I. Katsnelson, F. Withers, A. Mishchenko, L. Eaves, A. K. Geim, K. S. Novoselov, and A. Misra, Magnon-assisted tunnelling in van der Waals heterostructures based on $\mathrm{CrBr}_{3}$, Nat. Electron. 1, 344 (2018).

[12] D. R. Klein, D. MacNeill, J. L. Lado, D. Soriano, E. Navarro-Moratalla, K. Watanabe, T. Taniguchi, S. Manni, P. Canfield, J. Fernández-Rossier, and P. Jarillo-Herrero, Probing magnetism in 2D van der Waals crystalline insulators via electron tunneling, Science 360, 1218 (2018).

[13] Z. Wang, M. Gibertini, D. Dumcenco, T. Taniguchi, K. Watanabe, E. Giannini, and A. F. Morpurgo, Determining the phase diagram of atomically thin layered antiferromagnet $\mathrm{CrCl}_{3}$, Nat. Nanotechnol. 14, 1116 (2019).

[14] M. Wu, Z. Li, T. Cao, and S. G. Louie, Physical origin of giant excitonic and magneto-optical responses in twodimensional ferromagnetic insulators, Nat. Commun. 10, 2371 (2019).

[15] T. R. Paudel and E. Y. Tsymbal, Spin filtering in $\mathrm{CrI}_{3}$ tunnel junctions, ACS Appl. Mater. Interfaces 11, 15781 (2019).

[16] A. Molina-Sánchez, G. Catarina, D. Sangalli, and J. Fernández-Rossier, Magneto-optical response of chromium trihalide monolayers: Chemical trends, J. Mater. Chem. C 8, 8856 (2020).

[17] M. Kim, P. Kumaravadivel, J. Birkbeck, W. Kuang, S. G. $\mathrm{Xu}$, D. G. Hopkinson, J. Knolle, P. A. McClarty, A. I. Berdyugin, M. Ben Shalom, R. V. Gorbachev, S. J. Haigh,
S. Liu, J. H. Edgar, K. S. Novoselov, I. V. Grigorieva, and A. K. Geim, Micromagnetometry of two-dimensional ferromagnets, Nat. Electron. 2, 457 (2019).

[18] L. Thiel, Z. Wang, M. A. Tschudin, D. Rohner, I. GutiérrezLezama, N. Ubrig, M. Gibertini, E. Giannini, A. F. Morpurgo, and P. Maletinsky, Probing magnetism in 2D materials at the nanoscale with single-spin microscopy, Science 364, 973 (2019).

[19] D. A. Broadway, S. C. Scholten, C. Tan, N. Dontschuk, S. E. Lillie, B. C. Johnson, G. Zheng, Z. Wang, A. R. Oganov, S. Tian, C. Li, H. Lei, L. Wang, L. C. L. Hollenberg, and J. Tetienne, Imaging domain reversal in an ultrathin van der Waals ferromagnet, Adv. Mater. 32, 2003314 (2020).

[20] Q.-C. Sun, T. Song, E. Anderson, A. Brunner, J. Förster, T. Shalomayeva, T. Taniguchi, K. Watanabe, J. Gräfe, R. Stöhr, X. Xu, and J. Wrachtrup, Magnetic domains and domain wall pinning in atomically thin $\mathrm{CrBr}_{3}$ revealed by nanoscale imaging, Nat. Commun. 12, 1989 (2021).

[21] F. Fabre, A. Finco, A. Purbawati, A. Hadj-Azzem, N. Rougemaille, J. Coraux, I. Philip, and V. Jacques, Characterization of room-temperature in-plane magnetization in thin flakes of $\mathrm{CrTe}_{2}$ with a single-spin magnetometer, Phys. Rev. Mater. 5, 034008 (2021).

[22] J. Cenker, B. Huang, N. Suri, P. Thijssen, A. Miller, T. Song, T. Taniguchi, K. Watanabe, M. A. McGuire, D. Xiao, and X. Xu, Direct observation of two-dimensional magnons in atomically thin $\mathrm{CrI}_{3}$, Nat. Phys. 17, 20 (2021).

[23] M. Bałanda, AC susceptibility studies of phase transitions and magnetic relaxation: Conventional, molecular and lowdimensional magnets, Acta Phys. Pol. A 124, 964 (2013).

[24] C. V. Topping and S. J. Blundell, A.C. susceptibility as a probe of low-frequency magnetic dynamics, J. Phys.: Condens. Matter 31, 013001 (2019).

[25] M. Gibertini, M. Koperski, A. F. Morpurgo, and K. S. Novoselov, Magnetic 2D materials and heterostructures, Nat. Nanotechnol. 14, 408 (2019).

[26] S. Chikazumi, Physics of Ferromagnetism (Oxford University Press, Oxford, 1997), 2nd ed. Chapter 18

[27] I. Lovchinsky, J. D. Sanchez-Yamagishi, E. K. Urbach, S. Choi, S. Fang, T. I. Andersen, K. Watanabe, T. Taniguchi, A. Bylinskii, E. Kaxiras, P. Kim, H. Park, and M. D. Lukin, Magnetic resonance spectroscopy of an atomically thin material using a single-spin qubit, Science 355, 503 (2017).

[28] B. B. Zhou, P. C. Jerger, K.-H. Lee, M. Fukami, F. Mujid, J. Park, and D. D. Awschalom, Spatiotemporal Mapping of a Photocurrent Vortex in Monolayer $\mathrm{MoS}_{2}$ Using Diamond Quantum Sensors, Phys. Rev. X 10, 011003 (2020).

[29] S. Parkin and S.-H. Yang, Memory on the racetrack, Nat. Nanotechnol. 10, 195 (2015).

[30] S. Vélez, J. Schaab, M. S. Wörnle, M. Müller, E. Gradauskaite, P. Welter, C. Gutgsell, C. Nistor, C. L. Degen, M. Trassin, M. Fiebig, and P. Gambardella, Highspeed domain wall racetracks in a magnetic insulator, Nat. Commun. 10, 4750 (2019).

[31] J. Grollier, D. Querlioz, K. Y. Camsari, K. Everschor-Sitte, S. Fukami, and M. D. Stiles, Neuromorphic spintronics, Nat. Electron. 3, 360 (2020).

[32] M. Abramchuk, S. Jaszewski, K. R. Metz, G. B. Osterhoudt, Y. Wang, K. S. Burch, and F. Tafti, Controlling magnetic and optical properties of the van der Waals crystal 
$\mathrm{CrCl}_{3-x} \mathrm{Br}_{x}$ via mixed halide chemistry, Adv. Mater. 30, 1801325 (2018).

[33] T. A. Tartaglia, J. N. Tang, J. L. Lado, F. Bahrami, M. Abramchuk, G. T. McCandless, M. C. Doyle, K. S. Burch, Y. Ran, J. Y. Chan, and F. Tafti, Accessing new magnetic regimes by tuning the ligand spin-orbit coupling in van der Waals magnets, Sci. Adv. 6, eabb9379 (2020).

[34] H. H. Kim, B. Yang, S. Li, S. Jiang, C. Jin, Z. Tao, G. Nichols, F. Sfigakis, S. Zhong, C. Li, S. Tian, D. G. Cory, G.-X. Miao, J. Shan, K. F. Mak, H. Lei, K. Sun, L. Zhao, and A. W. Tsen, Evolution of interlayer and intralayer magnetism in three atomically thin chromium trihalides, Proc. Natl. Acad. Sci. 116, 11131 (2019).

[35] T. Hingant, J.-P. Tetienne, L. J. Martínez, K. Garcia, D. Ravelosona, J.-F. Roch, and V. Jacques, Measuring the Magnetic Moment Density in Patterned Ultrathin Ferromagnets with Submicrometer Resolution, Phys. Rev. Appl. 4, 014003 (2015).

[36] J.-S. Si, H. Li, B.-G. He, Z.-P. Cheng, and W.-B. Zhang, Revealing the underlying mechanisms of the stacking order and interlayer magnetism of bilayer $\mathrm{CrBr}_{3}$, J. Phys. Chem. C 125, 7314 (2021).

[37] C. L. Degen, F. Reinhard, and P. Cappellaro, Quantum sensing, Rev. Mod. Phys. 89, 035002 (2017).

[38] N. Aslam, M. Pfender, P. Neumann, R. Reuter, A. Zappe, F. Fávaro de Oliveira, A. Denisenko, H. Sumiya, S. Onoda, J. Isoya, and J. Wrachtrup, Nanoscale nuclear magnetic resonance with chemical resolution, Science 357, 67 (2017).

[39] D. R. Glenn, D. B. Bucher, J. Lee, M. D. Lukin, H. Park, and R. L. Walsworth, High-resolution magnetic resonance spectroscopy using a solid-state spin sensor, Nature $\mathbf{5 5 5}$, 351 (2018).

[40] F. Shi, Q. Zhang, P. Wang, H. Sun, J. Wang, X. Rong, M. Chen, C. Ju, F. Reinhard, H. Chen, J. Wrachtrup, J. Wang, and J. Du, Single-protein spin resonance spectroscopy under ambient conditions, Science 347, 1135 (2015).

[41] F. H. Salas and M. Mirabal-Garcia, Quenching of the Hopkinson maximum under contamination in the system Gd(0001)/W(110), Phys. Rev. B 41, 10859 (1990).

[42] K. Yang, W. Hu, H. Wu, M.-H. Whangbo, P. G. Radaelli, and A. Stroppa, Magneto-optical kerr switching properties of $\left(\mathrm{CrI}_{3}\right) 2$ and $\left(\mathrm{CrBr}_{3} / \mathrm{CrI}_{3}\right)$ bilayers, ACS Appl. Electron. Mater. 2, 1373 (2020).

[43] Y. Dovzhenko, F. Casola, S. Schlotter, T. X. Zhou, F. Büttner, R. L. Walsworth, G. S. D. Beach, and A. Yacoby, Magnetostatic twists in room-temperature skyrmions explored by nitrogen-vacancy center spin texture reconstruction, Nat. Commun. 9, 2712 (2018).

[44] O. Bostanjoglo and W. Vieweger, Magnetische bereiche in chromtrihalogeniden, Phys. Status Solidi B 39, 471 (1970).

[45] J. T. Ho and J. D. Litster, Divergences of the magnetic properties of $\mathrm{CrBr}_{3}$ near the critical point, J. Appl. Phys. 40, 1270 (1969).

[46] D. A. Wahab, M. Augustin, S. M. Valero, W. Kuang, S. Jenkins, E. Coronado, I. V. Grigorieva, I. J. Vera-Marun, E. Navarro-Moratalla, R. F. L. Evans, K. S. Novoselov, and E. J. G. Santos, Quantum rescaling, domain metastability, and hybrid domain-walls in $2 \mathrm{D} \mathrm{CrI}_{3}$ magnets, Adv. Mater. 33, 2004138 (2021).
[47] S. Tiwari, M. L. Van de Put, B. Sorée, and W. G. Vandenberghe, Critical behavior of the ferromagnets $\mathrm{CrI}_{3}$, $\mathrm{CrBr}_{3}$, and $\mathrm{CrGeTe}_{3}$ and the antiferromagnet $\mathrm{FeCl}_{2}: \mathrm{A}$ detailed first-principles study, Phys. Rev. B 103, 014432 (2021).

[48] N. Richter, D. Weber, F. Martin, N. Singh, U. Schwingenschlögl, B. V. Lotsch, and M. Kläui, Temperaturedependent magnetic anisotropy in the layered magnetic semiconductors $\mathrm{CrI}_{3}$ and $\mathrm{CrBr}_{3}$, Phys. Rev. Mater. 2, 024004 (2018).

[49] B. Kuhlow and M. Lambeck, Magnetic domain structures in $\mathrm{CrBr}_{3}$, Physica B+C 80, 365 (1975).

[50] Y. Liu and C. Petrovic, Anisotropic magnetocaloric effect in single crystals of $\mathrm{CrI}_{3}$, Phys. Rev. B 97, 174418 (2018).

[51] K. S. Novoselov, A. K. Geim, S. V. Dubonos, E. W. Hill, and I. V. Grigorieva, Subatomic movements of a domain wall in the Peierls potential, Nature 426, 812 (2003).

[52] T. R. Eichhorn, C. A. McLellan, and A. C. Bleszynski Jayich, Optimizing the formation of depth-confined nitrogen vacancy center spin ensembles in diamond for quantum sensing, Phys. Rev. Mater. 3, 113802 (2019).

[53] G. D. Fuchs, V. V. Dobrovitski, D. M. Toyli, F. J. Heremans, and D. D. Awschalom, Gigahertz dynamics of a strongly driven single quantum spin, Science 326, 1520 (2009).

[54] G. T. Rado, R. W. Wright, and W. H. Emerson, Ferromagnetism at very high frequencies. III. Two mechanisms of dispersion in a ferrite, Phys. Rev. 80, 273 (1950).

[55] M. Guyot and V. Cagan, Temperature dependence of the domain wall mobility in YIG, deduced from the frequency spectra of the initial susceptibility of polycrystals, J. Magn. Magn. Mater. 27, 202 (1982).

[56] E. Jedryka, R. J. Yeh, M. Ramesh, P. E. Wigen, and A. Pajaczkowska, Temperature dependence of domain wall resonance in $\mathrm{CrBr}_{3}$, J. Appl. Phys. 53, 8357 (1982).

[57] G. Infante, R. Varga, G. A. Badini-Confalonieri, and M. Vázquez, Locally induced domain wall damping in a thin magnetic wire, Appl. Phys. Lett. 95, 012503 (2009).

[58] Y. Romach, C. Müller, T. Unden, L. J. Rogers, T. Isoda, K. M. Itoh, M. Markham, A. Stacey, J. Meijer, S. Pezzagna, B. Naydenov, L. P. McGuinness, N. Bar-Gill, and F. Jelezko, Spectroscopy of Surface-Induced Noise Using Shallow Spins in Diamond, Phys. Rev. Lett. 114, 017601 (2015).

[59] X. Xi, Z. Wang, W. Zhao, J.-H. Park, K. T. Law, H. Berger, L. Forró, J. Shan, and K. F. Mak, Ising pairing in superconducting $\mathrm{NbSe}_{2}$ atomic layers, Nat. Phys. 12, 139 (2016).

[60] C. Xu, J. Feng, M. Kawamura, Y. Yamaji, Y. Nahas, S. Prokhorenko, Y. Qi, H. Xiang, and L. Bellaiche, Possible Kitaev Quantum Spin Liquid State in 2D Materials with $S=3 / 2$, Phys. Rev. Lett. 124, 087205 (2020).

[61] F.-S. Guo, B. M. Day, Y.-C. Chen, M.-L. Tong, A. Mansikkamäki, and R. A. Layfield, Magnetic hysteresis up to $80 \mathrm{~K}$ in a dysprosium metallocene single-molecule magnet, Science 362, 1400 (2018).

[62] M. M. Van De Loosdrecht, S. Draack, S. Waanders, J. G. Schlief, H. J. Krooshoop, T. Viereck, F. Ludwig, and B. Ten Haken, A novel characterization technique for 
superparamagnetic iron oxide nanoparticles: The superparamagnetic quantifier, compared with magnetic particle spectroscopy, Rev. Sci. Instrum. 90, 024101 (2019).

[63] A. Gao et al., Layer Hall effect in a 2D topological axion antiferromagnet, Nature 595, 521 (2021).

[64] J.-P. Tetienne, T. Hingant, L. Martínez, S. Rohart, A. Thiaville, L. H. Diez, K. Garcia, J.-P. Adam, J.-V. Kim, J.-F. Roch, I. Miron, G. Gaudin, L. Vila, B. Ocker, D. Ravelosona, and V. Jacques, The nature of domain walls in ultrathin ferromagnets revealed by scanning nanomagnetometry, Nat. Commun. 6, 6733 (2015).

[65] M. W. Doherty, J. Michl, F. Dolde, I. Jakobi, P. Neumann, N. B. Manson, and J. Wrachtrup, Measuring the defect structure orientation of a single NV- centre in diamond, New J. Phys. 16, 063067 (2014).

[66] D.-X. Chen, V. Skumryev, and H. Kronmüller, ac susceptibility of a spherical $\mathrm{Nd}_{2} \mathrm{Fe}_{14} \mathrm{~B}$ single crystal, Phys. Rev. B 46, 3496 (1992). 